\title{
A PRIORI ANISOTROPIC MESH ADAPTATION ON IMPLICITLY DEFINED SURFACES*
}

\author{
FRANCO DASSI ${ }^{\dagger}$, SIMONA PEROTTO $\ddagger$, AND LUCA FORMAGGIA
}

This work is dedicated to the memory of Dr. C. L. Lawson

\begin{abstract}
Mesh adaptation on surfaces demands particular care due to the important role played by the surface fitting. We propose an adaptive procedure based on a new error analysis which combines a rigorous anisotropic estimator for the $L^{1}$-norm of the interpolation error with an anisotropic heuristic control of the geometric error. We resort to a metric-based adaptive algorithm which employs local operations to modify the initial mesh according to the information provided by the error analysis. An extensive numerical validation corroborates the robustness of the error analysis as well as of the adaptive procedure.
\end{abstract}

Key words. anisotropic interpolation error estimates, metric-based mesh adaptation, finite elements on surfaces

AMS subject classifications. 65D05, 65N15, 65N50, 65N30

DOI. $10.1137 / 140995246$

1. Introduction and motivations. Mesh adaptation on surfaces is a topic of great interest in the scientific panorama due to its potential strong impact with a view to practical applications (see, e.g., [31, 33] among the most recent papers) and, more generally, to the approximation of partial differential equations on manifolds. Despite the relevance of this research field, there are still few works dealing with a surface mesh adaptation driven by a rigorous error analysis, and they are essentially confined to an isotropic context $[3,12,13,25,30]$.

In this paper we focus on implicitly defined surfaces $\Gamma \subset \mathbb{R}^{3}$ and we derive an anisotropic a priori error estimator to control the error associated with the approximation of a generic function $f \in H^{1}(\Gamma)$ via a piecewise linear quasi-interpolant operator. The triangular surface grid used to define the interpolant is also employed to approximate surface $\Gamma$. This choice leads us to include in the error analysis a contribution due to the geometric approximation in addition to the interpolation error. In this respect, we are consistent with the decomposition of the error provided in [12] in terms of a contribution related to the finite element approximation of $f$, a term associated with the geometric approximation of $\Gamma$ and a contribution due to data approximation. In particular, since we deal with an interpolation error analysis, we neglect the data error while identifying the discretization with the interpolation error.

The interest for an anisotropic setting is justified by the several applicative fields of interest in scientific computing (e.g., in biomedicine, geology, or aerodynamics),

* Submitted to the journal's Methods and Algorithms for Scientific Computing section November 10, 2014; accepted for publication (in revised form) September 11, 2015; published electronically November 17, 2015.

http://www.siam.org/journals/sisc/37-6/99524.html

$\dagger$ MOX, Dipartimento di Matematica, Politecnico di Milano, Piazza Leonardo da Vinci 32, I-20133, Milano, Italy. Current address: Weierstrass Institute for Applied Analysis and Stochastics, Berlin, Germany (franco.dassi@polimi.it).

${ }_{\ddagger}^{\ddagger}$ MOX, Dipartimento di Matematica, Politecnico di Milano, Piazza Leonardo da Vinci 32, I20133, Milano, Italy (simona.perotto@polimi.it, luca.formaggia@polimi.it). The second author work was supported by Project MIUR-PRIN 2010/2011, "Innovative Methods for Water Resources under Hydro-Climatic Uncertainty Scenarios." 
where phenomena may exhibit large variations along a certain direction with less significant changes along the other ones. In such cases, a standard isotropic adaptive algorithm may generate a very large number of elements to ensure a certain accuracy or, vice versa, may provide limited accuracy for a fixed number of elements. This is a well-established issue in the standard planar case (see, e.g., $[7,8,29,36])$ and it is expected to work analogously on surfaces, where the directionalities of the domain may be taken into account as well, via proper sizing, shaping, and orientation of the mesh elements.

The idea pursued in this work is to properly merge the interpolation analysis developed in [12] with the anisotropic setting proposed in [17] for a two-dimensional (2D) planar setting, and then successfully extended to a 3D volumetric framework in [15]. In particular, the derivation of an anisotropic counterpart of the Poincaré inequality represents the crucial result with a view to all anisotropic control of the interpolation error. Concerning the anisotropic bound for the geometric error, we move essentially from heuristic considerations.

The information provided by the global (interpolation plus geometric) error estimator is successively converted into a practical procedure to adapt the mesh via a metric-based approach, with the aim of reducing the number of mesh elements to guarantee a certain accuracy on the solution while equidistributing the error. To combine the interpolation with the geometric information, we propose three strategies. We use standard local mesh operations to change the initial (isotropic) mesh into the final adapted mesh matching the directional features of the function and of the surface. The employment of local operations is a novelty in the context of the anisotropic approach employed, e.g., in [17, 29], where a metric-based remeshing of the initial grid is essentially performed. Finally, we remark that the proposed approach due to the implicit representation of the surface relieves us from specific requirements on the surfaces of interest as, for instance, in [3,30].

The paper is organized as follows. In section 2 we settle the reference geometric setting and we introduce the basic ideas of the anisotropic framework. Section 3 represents the theoretical core of the paper providing the anisotropic estimator for the interpolation error and for the geometric error. In section 4 we tackle the crucial issue of merging the interpolation analysis with the geometric information. Section 5 provides an exhaustive numerical investigation, first by setting the adaptive procedure and then by validating it on both a closed and an open surface. The last section draws some conclusions starting from the numerical assessment.

2. The geometric setting. Let us consider a connected $C^{2}$-compact and orientable $2 \mathrm{D}$ surface $\Gamma$ embedded in $\mathbb{R}^{3}$. In particular, we adopt an implicit representation for $\Gamma$, i.e., we assume that $\Gamma$ coincides with the zero level set of a signed distance function $d: U_{0} \subset \mathbb{R}^{3} \rightarrow \mathbb{R}$, such that $d(\mathbf{x})=\operatorname{dist}(\mathbf{x}, \Gamma)$ for any $\mathbf{x} \in U_{0}$, and with $U_{0}$ an open subset of $\mathbb{R}^{3}$. In particular, if $\Gamma$ is a closed surface, we assume $d<0$ inside the volume enclosed by $\Gamma$, whereas $d>0$ outside, while if $\Gamma$ is an open surface, we assume $\partial \Gamma$ to be piecewise curvilinear in a sense that will be specified below.

Now, following [12], we assume that there exists a shell of width $\delta>0$ around $\Gamma$ given by $U_{\delta}=\left\{\mathbf{x} \in \mathbb{R}^{3}:|\operatorname{dist}(\mathbf{x})|<\delta\right\} \subset U_{0}$ (see Figure 1, left). In particular, the thickness $\delta$ of the shell is sufficiently small to guarantee the global uniqueness of the decomposition

$$
\mathbf{x}=a(\mathbf{x})+d(\mathbf{x}) \mathbf{n}(\mathbf{x}) \quad \forall \mathbf{x} \in U_{\delta}
$$

Copyright ( $)$ by SIAM. Unauthorized reproduction of this article is prohibited. 

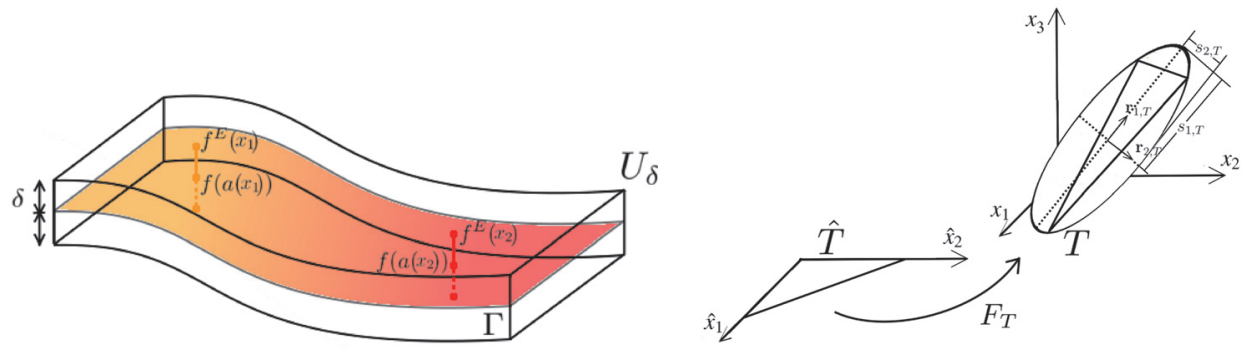

FIG. 1. Schematic representation of the shell $U_{\delta}$ and of the extended function $f^{E}$ (left); geometric interpretation of map $F_{T}$ and of the main anisotropic quantities (right).

with $a: U_{\delta} \rightarrow \Gamma$ the orthogonal projection operator onto $\Gamma$ and $\mathbf{n}(\mathbf{x})=\nabla d(\mathbf{x})$ the outward unit normal to $\Gamma$ at $\mathbf{x}$. More details about the choice of $\delta$ can be found, e.g., in [12]. The projection $a$ is instrumental in extending the definition of a function $f$ assigned on $\Gamma$ to the whole shell $U_{\delta}$, thanks to the following relation:

$$
f^{E}(\mathbf{x})=f(a(\mathbf{x})) \quad \forall \mathbf{x} \in U_{\delta} .
$$

Essentially, the extended function $f^{E}$ can be identified with the extension along wires of the function $f$ (see Figure 1, left). Via $f^{E}$, we define the tangential gradient $\nabla_{\Gamma} f$ on $\Gamma$ of the function $f: \Gamma \rightarrow \mathbb{R}$ as

$$
\nabla_{\Gamma} f=\nabla f^{E}-\left(\mathbf{n} \cdot \nabla f^{E}\right) \mathbf{n}
$$

with $\nabla$ the standard gradient operator in $\mathbb{R}^{3}$. Thanks to (2.2), the tangential gradient $\nabla_{\Gamma} f$ depends only on the values of $f$ on $\Gamma$ despite (2.3) involving the whole shell $U_{\delta}$.

Now, in view of practical computations, we replace surface $\Gamma$ with a polyhedral surface $\Gamma_{h} \subset U_{\delta}$ consisting of a set $\mathcal{T}_{h}=\{T\}$ of triangular faces $T$ of diameter $h_{T}$, such that $\Gamma_{h} \equiv \cup_{T \in \mathcal{T}_{h}} T$. We denote by $\mathcal{V}$ the set of the vertices of $\Gamma_{h}$ and we demand $\mathcal{V} \subset \Gamma$. Let $\mathbf{n}_{h}$ be the piecewise constant unit outer normal to $\Gamma_{h}$. We assume $\mathbf{n} \cdot \mathbf{n}_{h}>0$ everywhere on $\Gamma_{h}$. Moreover, since $\Gamma_{h} \subset U_{\delta}$, we employ the orthogonal projection defined in (2.1) to relate $\Gamma_{h}$ with $\Gamma$ as well, by demanding $a: \Gamma_{h} \rightarrow \Gamma$ to be bijective. The Jacobian associated with such a bijection is denoted by $\mu_{h}$, i.e., we have that

$$
\mu_{h}(\mathbf{x}) d \Gamma_{h}(\mathbf{x})=d \Gamma(a(\mathbf{x})) \quad \forall \mathbf{x} \in \Gamma_{h} .
$$

Finally, if $\Gamma$ is an open surface, we require $\partial \Gamma \equiv a\left(\partial \Gamma_{h}\right)$. This implies assuming $\partial \Gamma$ piecewise linear, so that we are relieved from the error due to the approximation of $\partial \Gamma$.

Analogously, any function $f$ is replaced by a discrete counterpart $f_{h}$. For this purpose, we introduce the space $X_{h}$ of the continuous functions which are affine on each face $T$ of $\Gamma_{h}$ [13] and we approximate $f$ with a function $f_{h} \in X_{h}$ to be properly defined. We denote by $\varphi_{\mathbf{z}_{i}} \in X_{h}$ the standard basis function associated with the vertex $\mathbf{z}_{i} \in \mathcal{V}$, such that $\varphi_{\mathbf{z}_{i}}\left(\mathbf{z}_{j}\right)=\delta_{i j}$, for any $\mathbf{z}_{i}, \mathbf{z}_{j} \in \mathcal{V}$, with $\delta_{i j}$ the Kronecker symbol.

2.1. Source of the anisotropic information. To uniquely define the size, according to an isotropic approach, together with the shape and the orientation of each 
face $T$ of the mesh, we extend the approach proposed in [17] for a 2D planar setting to a nonplanar framework. We choose as a source for the anisotropic information the invertible affine map $F_{T}$ from a reference planar triangle $\widehat{T} \subset \mathbb{R}^{2}$ to the general (nondegenerate) triangle $T \in \mathcal{T}_{h}$ embedded in $\mathbb{R}^{3}$ (see Figure 1, right). The map $F_{T}: \widehat{T} \rightarrow T$ is defined by

$$
\mathbf{x}=\left(x_{1}, x_{2}, x_{3}\right)^{\prime}=F_{T}(\widehat{\mathbf{x}})=M_{T} \widehat{\mathbf{x}}+\mathbf{b}_{T} \in T
$$

with $\widehat{\mathbf{x}}=\left(\widehat{x}_{1}, \widehat{x}_{2}\right)^{\prime} \in \widehat{T}, M_{T} \in \mathbb{R}^{3 \times 2}$, and $\mathbf{b}_{T} \in \mathbb{R}^{3}$. As reference element $\widehat{T}$ we select the triangle with vertices $(0,0),(1,0),(0,1)$. For this choice, we have $\left[M_{T}\right]_{i j}=$ $v_{i}^{j+1}-v_{i}^{1},\left[b_{T}\right]_{j}=v_{j}^{1}$, for $i=1,2,3, j=1,2$, and where $\mathbf{v}^{k}=\left(v_{1}^{k}, v_{2}^{k}, v_{3}^{k}\right)^{\prime}$, for $k=1,2,3$, denotes the $k$ th vertex of $T$.

The anisotropic information of each triangle $T$ is derived from the singular value decomposition (SVD) of $M_{T}$ given by $M_{T}=U_{T} \Sigma_{T} V_{T}^{\prime}$, where $U_{T} \in \mathbb{R}^{3 \times 3}$ and $V_{T} \in \mathbb{R}^{2 \times 2}$ are unitary matrices, while $\Sigma_{T}=\operatorname{diag}\left(s_{1, T}, s_{2, T}\right) \in \mathbb{R}^{3 \times 2}$ is a rectangular diagonal matrix with nonnegative real entries representing the singular values of $M_{T}$ [23]. The columns of the matrices $U_{T}=\left[\mathbf{r}_{1, T}, \mathbf{r}_{2, T}, \mathbf{n}_{h}\right]$ and $V_{T}$ collect the left- and right-singular vectors of $M_{T}$, respectively. The SVD of $M_{T}$ allows us to completely characterize the triangle $T \in \mathcal{T}_{h}$. The singular values of $M_{T}$ measure the lengths of the semi-axes of the ellipse circumscribing $T$ and lying on the plane identified by $T$, while the singular vectors $\mathbf{r}_{1, T}$ and $\mathbf{r}_{2, T}$ provide the directions of these semi-axes (see Figure 1, right). Without loss of generality, henceforth we assume $s_{1, T} \geq s_{2, T}$. Moreover, we introduce the aspect ratio $\sigma_{T}=s_{1, T} / s_{2, T}$, which quantifies the deformation of $T$. The aspect ratio is always greater than or equal to one, with $\sigma_{T}=1$ when $T$ coincides with an equilateral triangular face.

In view of a mesh adaptation procedure, we define a suitable interpolation operator, by adopting a standard notation for both the Lebesgue and Sobolev spaces of functions defined on surfaces as well as for the associated norms (see, e.g., [13]). Following [12], given a function $f: \Gamma \rightarrow \mathbb{R}$ such that $f \in L^{1}(\Gamma)$, we first introduce the averaged nodal values

$$
f_{\mathbf{z}}^{E}=\frac{1}{\int_{\omega_{\mathbf{z}}} \varphi_{\mathbf{z}} d \omega_{\mathbf{z}}} \int_{\omega_{\mathbf{z}}} \varphi_{\mathbf{z}} f^{E} d \omega_{\mathbf{z}} \quad \forall \mathbf{z} \in \mathcal{V}
$$

where $\omega_{\mathbf{z}}$ is the patch of the faces in $\Gamma_{h}$ sharing vertex $\mathbf{z}, f^{E}$ is the extension of the function $f$ to $U_{\delta}$ according to $(2.2)$, and $\varphi_{\mathbf{z}}$ is the basis function associated with vertex $\mathbf{z}$, with support $\omega_{\mathbf{z}}$. Thus, the interpolant we are interested in is given by

$$
I_{h} f^{E}(\mathbf{x})=\sum_{\mathbf{z} \in \mathcal{V}} f_{\mathbf{z}}^{E} \varphi_{\mathbf{z}}(\mathbf{x}) \quad \forall f \in L^{1}(\Gamma) .
$$

Actually, $I_{h}$ is a quasi-interpolant operator, similar to the Clément or Scott-Zhang interpolants $[9,35]$ (see also, e.g., $[3,16,17]$ ). Moreover, since functions $\left\{\varphi_{\mathbf{z}}\right\}_{\mathbf{z} \in \mathcal{V}}$ constitute a partition of unity, the $L^{1}\left(\Gamma_{h}\right)$-norm of the interpolation error is identically equal to zero, i.e.,

$$
\int_{\Gamma_{h}}\left(f^{E}-I_{h} f^{E}\right) d \Gamma_{h}=\sum_{\mathbf{z} \in \mathcal{V}} \int_{\omega_{\mathbf{z}}}\left(f^{E}-f_{\mathbf{z}}^{E}\right) \varphi_{\mathbf{z}} d \omega_{\mathbf{z}}=0,
$$

where $\operatorname{supp}\left(\varphi_{\mathbf{z}}\right)=\omega_{\mathbf{z}}$. In what follows, we identify the approximation $f_{h} \in X_{h}$ for the generic function $f$ defined on $\Gamma$ with the interpolant in (2.7). In particular, an anisotropic control for the $L^{1}(\Gamma)$-norm of the error $f-I_{h} f^{E}$ will drive the mesh adaptive procedure. 
3. Anisotropic error estimates. In this section we provide the theoretical tool at the basis of the proposed adaptive procedure. We exploit the standard decomposition of the error into a contribution related to the finite element approximation and a contribution associated with the geometric approximation of the surface $\Gamma$ via $\Gamma_{h}$ [12]. Since we deal with an interpolation error analysis, we neglect the error associated with the data approximation, and we identify the discretization with the interpolation error.

3.1. The interpolation error. To derive an anisotropic bound for the interpolation error $f-I_{h} f^{E}$ associated with the operator (2.7), we preliminarily prove the following result, which generalizes Lemma 2.2 in [12] to an anisotropic context.

Lemma 3.1 (anisotropic Poincaré inequality). Let $f \in H^{1}(\Gamma)$. Then, for each node $\mathbf{z} \in \mathcal{V}$, there exists a constant $C$ such that

$$
\left\|f^{E}-f_{\mathbf{z}}^{E}\right\|_{L^{2}\left(\omega_{\mathbf{z}}\right)} \leq C\left[\sum_{T \in \omega_{\mathbf{z}}}\left(\sum_{i=1}^{2} s_{i, T}^{2} \mathbf{r}_{i, T}^{\prime} G_{T}\left(f^{E}\right) \mathbf{r}_{i, T}\right)\right]^{1 / 2}
$$

with $f^{E}: U_{\delta} \rightarrow \mathbb{R}$ the extension of $f$ to $U_{\delta}$ according to (2.2) and $f_{\mathbf{z}}^{E}$ defined as in (2.6), and where $G_{T}$ is the symmetric positive semidefinite matrix given by

$$
G_{T}\left(f^{E}\right)=\left[\begin{array}{ccc}
\int_{T}\left(g_{1}\right)^{2} d T & \int_{T} g_{1} g_{2} d T & \int_{T} g_{1} g_{3} d T \\
\int_{T}^{g_{1} g_{2} d T} & \int_{T}\left(g_{2}\right)^{2} d T & \int_{T} g_{2} g_{3} d T \\
\int_{T}^{g_{1} g_{3} d T} & \int_{T}^{g_{2} g_{3} d T} & \int_{T}\left(g_{3}\right)^{2} d T
\end{array}\right],
$$

where $g_{i}=\left(\nabla_{\Gamma_{h}} f^{E}\right)_{i}$, for $i=1,2,3$, is the $i$ th component of the tangential gradient $\nabla_{\Gamma_{h}} f^{E}=\nabla f^{E}-\left(\mathbf{n}_{h} \cdot \nabla f^{E}\right) \mathbf{n}_{h}$ with respect to the standard Cartesian coordinate system in $\mathbb{R}^{3}$.

Proof. The first part of this proof follows the proof of Lemma 2.2 in [12]. Thanks to definition (2.6) and the Cauchy-Schwarz inequality, we first get

$$
\left\|f_{\mathbf{z}}^{E}\right\|_{L^{2}\left(\omega_{\mathbf{z}}\right)}=\left|\omega_{\mathbf{z}}\right|^{1 / 2}\left|f_{\mathbf{z}}^{E}\right| \leq\left|\omega_{\mathbf{z}}\right|^{1 / 2} \frac{\left\|\varphi_{\mathbf{z}}\right\|_{L^{2}\left(\omega_{\mathbf{z}}\right)}}{\left\|\varphi_{\mathbf{z}}\right\|_{L^{1}\left(\omega_{\mathbf{z}}\right)}}\left\|f^{E}\right\|_{L^{2}\left(\omega_{\mathbf{z}}\right)}
$$

with $|\varpi|$ the measure of a generic set $\varpi \subset \mathbb{R}^{d}$ for $d=1,2,3$. Now, by exploiting the map $F_{T}$ in (2.5), for each $T \in \omega_{\mathbf{z}}$, we consider the piecewise affine map $F_{\mathbf{z}}: \widehat{\omega}_{\mathbf{z}} \rightarrow \omega_{\mathbf{z}}$, where $\widehat{\omega}_{\mathbf{z}}$ is the union of the inverse image $F_{T}^{-1}(T)$ of all the triangles $T$ constituting $\omega_{\mathbf{z}}$. Analogously, we denote by $\widehat{u}$ the inverse image of a generic function $u \in H^{1}\left(\Gamma_{h}\right)$ via $F_{T}$. The $L^{2}\left(\omega_{\mathbf{z}}\right)$ - and $L^{1}\left(\omega_{\mathbf{z}}\right)$-norms in (3.3) can be computed coming back to the reference framework as

$$
\left\|\varphi_{\mathbf{z}}\right\|_{L^{p}\left(\omega_{\mathbf{z}}\right)}^{p}=\sum_{T \in \omega_{\mathbf{z}}} \int_{T}\left(\varphi_{\mathbf{z}}\right)^{p} d T=\sum_{T \in \omega_{\mathbf{z}}} \frac{|T|}{|\widehat{T}|} \int_{\widehat{T}}\left(\widehat{\varphi}_{\mathbf{z}}\right)^{p} d \widehat{T}=\left|\omega_{\mathbf{z}}\right| \frac{\int_{\widehat{T}}\left(\widehat{\varphi}_{\mathbf{z}}\right)^{p} d \widehat{T}}{|\widehat{T}|}
$$

where $\left\|\widehat{\varphi}_{\mathbf{z}}\right\|_{L^{1}(\widehat{T})}=1 / 6,\left\|\widehat{\varphi}_{\mathbf{z}}\right\|_{L^{2}(\widehat{T})}=1 / \sqrt{12}$, and $|\widehat{T}|=1 / 2$. By substituting (3.4) in (3.3), we get $\left\|f_{\mathbf{z}}^{E}\right\|_{L^{2}\left(\omega_{\mathbf{z}}\right)} \leq \sqrt{3 / 2}\left\|f^{E}\right\|_{L^{2}\left(\omega_{\mathbf{z}}\right)}$. In a similar way, for a constant $K \in \mathbb{R}$ 
and thanks to definition (2.6) and to the Cauchy-Schwarz inequality, we have

$$
\begin{aligned}
\left\|f_{\mathbf{z}}^{E}-K\right\|_{L^{2}\left(\omega_{\mathbf{z}}\right)} & =\left|\omega_{\mathbf{z}}\right|^{1 / 2}\left|f_{\mathbf{z}}^{E}-K\right|=\frac{\left|\omega_{\mathbf{z}}\right|^{1 / 2}}{\left|\int_{\omega_{\mathbf{z}}} \varphi_{\mathbf{z}} d \omega_{\mathbf{z}}\right|}\left|\int_{\omega_{\mathbf{z}}}\left(f^{E}-K\right) \varphi_{\mathbf{z}} d \omega_{\mathbf{z}}\right| \\
& \leq \frac{\left|\omega_{\mathbf{z}}\right|^{1 / 2}}{\left\|\varphi_{\mathbf{z}}\right\|_{L^{1}\left(\omega_{\mathbf{z}}\right)}}\left\|f^{E}-K\right\|_{L^{2}\left(\omega_{\mathbf{z}}\right)}\left\|\varphi_{\mathbf{z}}\right\|_{L^{2}\left(\omega_{\mathbf{z}}\right)} \leq \sqrt{\frac{3}{2}}\left\|f^{E}-K\right\|_{L^{2}\left(\omega_{\mathbf{z}}\right)} .
\end{aligned}
$$

The triangle inequality immediately yields

$$
\left\|f^{E}-f_{\mathbf{z}}^{E}\right\|_{L^{2}\left(\omega_{\mathbf{z}}\right)} \leq\left(1+\sqrt{\frac{3}{2}}\right)\left\|f^{E}-K\right\|_{L^{2}\left(\omega_{\mathbf{z}}\right)} .
$$

Now, the idea is to properly select $K$ and to exploit the spectral decomposition for $M_{T}$ to obtain an anisotropic bound for the right-hand side in (3.5). We choose $K=|\widehat{T}|^{-1} \int_{\widehat{T}} \eta(\widehat{\mathbf{x}}) d \widehat{T}$, where $\eta$ is a function defined on $\widehat{T}$ such that $\eta(\widehat{\mathbf{x}})=f^{E}\left(F_{T}(\widehat{\mathbf{x}})\right)$ for any $\widehat{\mathbf{x}} \in \widehat{T}$. Thus, thanks to the standard Poincaré inequality, we have

$$
\left\|f^{E}-f_{\mathbf{z}}^{E}\right\|_{L^{2}\left(\omega_{\mathbf{z}}\right)}^{2} \leq C \sum_{T \in \omega_{\mathbf{z}}} \frac{|T|}{|\widehat{T}|} \int_{\widehat{T}}(\eta-K)^{2} d \widehat{T} \leq C \sum_{T \in \omega_{\mathbf{z}}} \frac{|T|}{|\widehat{T}|} \int_{\widehat{T}}(\widehat{\nabla} \eta)^{2} d \widehat{T}
$$

with $\widehat{\nabla}$ the gradient operator associated with the coordinate system $\left(\widehat{x}_{1}, \widehat{x}_{2}\right)$ in the reference setting and where $C$ does include the constant value in (3.5) and the Poincaré constant as well. We remark that $\widehat{\nabla} \eta=M_{T}^{\prime} \nabla_{\Gamma_{h}} f^{E}$. This yields, coming back to $\Gamma_{h}$, that

$$
\left\|f^{E}-f_{\mathbf{z}}^{E}\right\|_{L^{2}\left(\omega_{\mathbf{z}}\right)}^{2} \leq C \sum_{T \in \omega_{\mathbf{z}}} \int_{T}\left|M_{T}^{\prime} \nabla_{\Gamma_{h}} f^{E}\right|^{2} d T
$$

To introduce the anisotropic information, we resort to the SVD of $M_{T}=U_{T} \Sigma_{T} V_{T}^{\prime}$ to have

$$
\left\|f^{E}-f_{\mathbf{z}}^{E}\right\|_{L^{2}\left(\omega_{\mathbf{z}}\right)}^{2} \leq C \sum_{T \in \omega_{\mathbf{z}}} \int_{T}\left[\left(\nabla_{\Gamma_{h}} f^{E}\right)^{\prime} U_{T} \Sigma_{T} \Sigma_{T}^{\prime} U_{T}^{\prime}\left(\nabla_{\Gamma_{h}} f^{E}\right)\right] d T
$$

where matrix $V_{T}$ does not provide any contribution since $V_{T}^{\prime} V_{T}=I$. Now, the product $U_{T} \Sigma_{T} \Sigma_{T}^{\prime} U_{T}^{\prime}$ in (3.6) can be easily expressed in terms of the anisotropic lengths $s_{i, T}$ and directions $\mathbf{r}_{i, T}$ as $U_{T} \Sigma_{T} \Sigma_{T}^{\prime} U_{T}^{\prime}=\sum_{i=1}^{2} s_{i, T}^{2} \mathbf{r}_{i, T} \otimes \mathbf{r}_{i, T}, \otimes$ denoting the standard outer product between vectors. This leads to rewriting (3.6) as

$$
\left\|f^{E}-f_{\mathbf{z}}^{E}\right\|_{L^{2}\left(\omega_{\mathbf{z}}\right)}^{2} \leq C \sum_{T \in \omega_{\mathbf{z}}}\left[\sum_{i=1}^{2} s_{i, T}^{2} \int_{T}\left(\nabla_{\Gamma_{h}} f^{E}\right)^{\prime} \mathbf{r}_{i, T} \otimes \mathbf{r}_{i, T}\left(\nabla_{\Gamma_{h}} f^{E}\right) d T\right] .
$$

Straightforward algebraic manipulations show that

$$
\left(\nabla_{\Gamma_{h}} f^{E}\right)^{\prime} \mathbf{r}_{i, T} \otimes \mathbf{r}_{i, T}\left(\nabla_{\Gamma_{h}} f^{E}\right)=\mathbf{r}_{i, T}^{\prime} \nabla_{\Gamma_{h}} f^{E} \otimes \nabla_{\Gamma_{h}} f^{E} \mathbf{r}_{i, T}=\mathbf{r}_{i, T}^{\prime} G_{T}\left(f^{E}\right) \mathbf{r}_{i, T}
$$

with $G_{T}$ defined as in (3.2). This completes the proof.

Remark 3.1. The quantity $C$ in (3.1) does not depend on any geometric feature nor on the regularity of $f$. Moreover, the introduction of the shell $U_{\delta}$ containing the 
surface of interest allows us to manage the tangential gradient as a standard threedimensional entity. This device justifies that the matrix (3.2) has a rank at most equal to two.

As expected, the anisotropic estimate (3.1) provides as a particular case the corresponding isotropic inequality (2.2.29) in [12] by exhibiting a more complex structure with respect to the isotropic result. The diameter $h_{\mathbf{z}}$ of the patch characterizing the isotropic estimate is replaced by the anisotropic lengths $s_{i, T}$ with $i=1,2$. Likewise, the first order derivatives involved in the $L^{2}\left(\omega_{\mathbf{z}}\right)$-norm of $\nabla_{\Gamma_{h}} f^{E}$ in the isotropic case are now projected along the anisotropic directions $\mathbf{r}_{i, T}$, with $i=1,2$, via the products $\mathbf{r}_{i, T}^{\prime} G_{T}\left(f^{E}\right) \mathbf{r}_{i, T}$. The intrinsic potentiality of estimate (3.1) is more evident by rewriting it in terms of the aspect ratio $\sigma_{T}$ as

$$
\left\|f^{E}-f_{\mathbf{z}}^{E}\right\|_{L^{2}\left(\omega_{\mathbf{z}}\right)} \leq C\left[\sum_{T \in \omega_{\mathbf{z}}}|T|\left(\sigma_{T} \mathbf{r}_{1, T}^{\prime} G_{T}\left(f^{E}\right) \mathbf{r}_{1, T}+\frac{1}{\sigma_{T}} \mathbf{r}_{2, T}^{\prime} G_{T}\left(f^{E}\right) \mathbf{r}_{2, T}\right)\right]^{1 / 2}
$$

where we have exploited the relation $|T|=|\widehat{T}| s_{1, T} s_{2, T}$ by including the area $|\widehat{T}|$ into $C$. Thus, $|T|$ provides the information associated with the size of $T$, the shape of $T$ is identified by the aspect ratio $\sigma_{T}$, whereas the orientation of $T$ is fixed by the directions $\mathbf{r}_{1, T}$ and $\mathbf{r}_{2, T}$.

The next result represents the main theoretical statement of this paper.

Proposition 3.2. Let $f \in H^{1}(\Gamma)$ and let $f^{E}: U_{\delta} \rightarrow \mathbb{R}$ be the extension of $f$ to $U_{\delta}$ according to (2.2). Then, there exists a constant $C$ such that

$$
\left\|f-I_{h} f^{E}\right\|_{L^{1}(\Gamma)} \leq C \sum_{T \in \mathcal{T}_{h}}|T|^{1 / 2} \alpha_{T} \nu_{T}\left(\sigma_{T}, \mathbf{r}_{1, T}, f^{E}\right),
$$

where $\alpha_{T}=\sum_{\mathbf{z} \in T}\left\|\varphi_{\mathbf{z}} \mu_{h}\right\|_{L^{2}\left(\omega_{\mathbf{z}}\right)}$, with $\mu_{h}$ defined as in (2.4) and $\varphi_{\mathbf{z}}$ the basis function associated with vertex $\mathbf{z}$, while

$$
\nu_{T}\left(\sigma_{T}, \mathbf{r}_{1, T}, f^{E}\right)=\left(\sigma_{T} \mathbf{r}_{1, T}^{\prime} G_{T}\left(f^{E}\right) \mathbf{r}_{1, T}+\frac{1}{\sigma_{T}} \mathbf{r}_{2, T}^{\prime} G_{T}\left(f^{E}\right) \mathbf{r}_{2, T}\right)^{1 / 2}
$$

with $G_{T}$ the matrix in (3.2).

Proof. By employing the Jacobian $\mu_{h}$ in (2.4), definition (2.7), and the partition of unity property characterizing the set $\left\{\varphi_{\mathbf{z}}\right\}_{\mathbf{z} \in \mathcal{V}}$, we get

$$
\begin{gathered}
\left\|f-I_{h} f^{E}\right\|_{L^{1}(\Gamma)}=\int_{\Gamma}\left|f(\mathbf{x})-I_{h} f^{E}(\mathbf{x})\right| d \Gamma=\int_{\Gamma_{h}}\left|f^{E}(\mathbf{x})-I_{h} f^{E}(\mathbf{x})\right|\left|\mu_{h}(\mathbf{x})\right| d \Gamma_{h} \\
=\int_{\Gamma_{h}}\left|\sum_{\mathbf{z} \in \mathcal{V}}\left(f^{E}(\mathbf{x})-f_{\mathbf{z}}^{E}\right) \varphi_{\mathbf{z}}(\mathbf{x})\right|\left|\mu_{h}(\mathbf{x})\right| d \Gamma_{h} \leq \sum_{\mathbf{z} \in \mathcal{V}} \int_{\omega_{\mathbf{z}}}\left|f^{E}(\mathbf{x})-f_{\mathbf{z}}^{E}\right|\left|\varphi_{\mathbf{z}}(\mathbf{x}) \mu_{h}(\mathbf{x})\right| d \omega_{\mathbf{z}},
\end{gathered}
$$

where the localization of the integral on $\Gamma_{h}$ to $\omega_{\mathbf{z}}$ is due to the support of $\varphi_{\mathbf{z}}$. Via the Cauchy-Schwarz inequality and thanks to estimate (3.7), we derive

$$
\begin{aligned}
& \left\|f-I_{h} f^{E}\right\|_{L^{1}(\Gamma)} \leq \sum_{\mathbf{z} \in \mathcal{V}}\left\|f^{E}-f_{\mathbf{z}}^{E}\right\|_{L^{2}\left(\omega_{\mathbf{z}}\right)}\left\|\varphi_{\mathbf{z}} \mu_{h}\right\|_{L^{2}\left(\omega_{\mathbf{z}}\right)} \\
\leq & C \sum_{\mathbf{z} \in \mathcal{V}}\left\|\varphi_{\mathbf{z}} \mu_{h}\right\|_{L^{2}\left(\omega_{\mathbf{z}}\right)}\left[\sum_{T \in \omega_{\mathbf{z}}}|T|\left(\sigma_{T} \mathbf{r}_{1, T}^{\prime} G_{T}\left(f^{E}\right) \mathbf{r}_{1, T}+\frac{1}{\sigma_{T}} \mathbf{r}_{2, T}^{\prime} G_{T}\left(f^{E}\right) \mathbf{r}_{2, T}\right)\right]^{1 / 2} .
\end{aligned}
$$

A reordering of the terms leads to the final result.

Remark 3.2. The choice of the $L^{1}(\Gamma)$-norm to estimate the interpolation error is not so recurrent in the literature (see, e.g., $[5,1]$ ). Indeed, the $L^{2}(\Gamma)-$ and the 
$H^{1}(\Gamma)$-norms are usually employed, both in an isotropic and in an anisotropic context (see, e.g., $[37,2,17,21,13]$ ). Nevertheless, as is evident from the proof of estimate (3.8), the $L^{1}(\Gamma)$-norm allows us to exploit the anisotropic Poincaré inequality in a straightforward way to bound the interpolation error. Moreover, the choice of the $L^{1}(\Gamma)$-norm will be instrumental with a view to an a posteriori error analysis, as in $[12]$.

3.2. The geometric error. We are now interested in controlling the error due to the fitting of the surface $\Gamma$ via $\Gamma_{h}$. The estimate we propose is heuristic. In more detail, to quantify the mismatch between $\Gamma$ and $\Gamma_{h}$, we resort to the signed distance function $d$ whose zero level set coincides with $\Gamma$. The employment of the orthogonal projection $a(\cdot)$ in (2.1) to relate $\Gamma$ and $\Gamma_{h}$ via a bijection suggests we identify the geometric error with the quantity $\left\|d-I_{h} d\right\|_{L^{1}(\Gamma)}$, after assuming sufficient regularity on $d$. In some sense, we are supposing that the discrete surface $\Gamma_{h}$ coincides with the zero level set of the function $I_{h} d$. Such an identification immediately leads us to provide an anisotropic estimate for the geometric error, simply by particularizing Proposition 3.2 to the distance function.

Proposition 3.3. Let $d: U_{0} \rightarrow \mathbb{R}$ be the signed distance function associated with the implicit representation of $\Gamma$ and let $d \in H^{1}\left(U_{0}\right)$. Then, there exists a constant $C$ such that

$$
\left\|d-I_{h} d\right\|_{L^{1}(\Gamma)} \leq C \sum_{T \in \mathcal{T}_{h}}|T|^{1 / 2} \alpha_{T} \nu_{T}\left(\sigma_{T}, \mathbf{r}_{1, T}, d\right)
$$

with $\alpha_{T}$ and $\nu_{T}\left(\sigma_{T}, \mathbf{r}_{1, T}, d\right)$ defined according to Proposition 3.2.

By comparing estimates (3.8) and (3.9), we remark that function $d$ does not require any extension via operator $a(\cdot)$, since it is defined directly on the whole shell $U_{\delta}$. Moreover, the regularity demanded on $d$ is guaranteed for smooth surfaces as the ones in section 5.2. The choice of the distance function may clearly affect the distribution of the geometric error. We choose $d$ as the Euclidean distance, in accordance with the definition of implicit surface. Other examples of geometric error control are available in the literature (see, e.g., $[12,19]$ ).

Moving from Propositions 3.2 and 3.3, we define the a priori anisotropic error estimators

$$
\eta_{I}=\sum_{T \in \mathcal{T}_{h}} \eta_{I, T}, \quad \eta_{G}=\sum_{T \in \mathcal{T}_{h}} \eta_{G, T}
$$

where $\eta_{I, T}=|T|^{1 / 2} \alpha_{T} \nu_{T}\left(\sigma_{T}, \mathbf{r}_{1, T}, f^{E}\right), \eta_{G, T}=|T|^{1 / 2} \alpha_{T} \nu_{T}\left(\sigma_{T}, \mathbf{r}_{1, T}, d\right)$, to control the interpolation and the geometric error, respectively. The two estimators share the same structure and both depend on the anisotropic geometric quantities. In section 5.2 , we will numerically investigate the convergence rate of $\eta_{I}$ and $\eta_{G}$, separately.

4. Merging the interpolation with the geometric error. The goal of this section is twofold. First, we focus on a method to commute, separately, estimators $\eta_{I}$ and $\eta_{G}$ into an operative procedure to anisotropically adapt the mesh $\mathcal{T}_{h}$. Successively, we consider different techniques to merge the information provided by the two estimators. Actually, the global estimator $\eta_{I G}=\eta_{I}+\eta_{G}$ will be employed only to estimate the global error. To generate the adapted mesh, we resort to a more intrinsic way via the concept of metric $[20,27]$.

4.1. From the estimator to the metric. A metric associated with the surface $\Gamma \subset \mathbb{R}^{3}$ is a symmetric positive semidefinite tensor $\mathcal{M}_{\Gamma}: \Gamma \rightarrow \mathbb{R}^{3 \times 3}$ identified, for each 
point $\mathbf{x} \in \Gamma$, by two strictly positive scalar functions $\rho_{1}=\rho_{1}(\mathbf{x})$ and $\rho_{2}=\rho_{2}(\mathbf{x})$ and by three vector functions $\mathbf{u}_{i}=\mathbf{u}_{i}(\mathbf{x}) \in \mathbb{R}^{3}$ of unitary norm, such that $\mathbf{u}_{i}(\mathbf{x}) \cdot \mathbf{u}_{j}(\mathbf{x})=$ $\delta_{i j}$, for any $\mathbf{x} \in \Gamma$ and for $i, j=1,2,3$. Thus, $\mathcal{M}_{\Gamma}(\mathbf{x})=U^{\prime}(\mathbf{x}) R^{-2}(\mathbf{x}) U(\mathbf{x})$, with $U(\mathbf{x})=\left[\begin{array}{lll}\mathbf{u}_{1}(\mathbf{x}) & \mathbf{u}_{2}(\mathbf{x}) & \mathbf{u}_{3}(\mathbf{x})\end{array}\right]$ and $R^{-2}(\mathbf{x})=\operatorname{diag}\left(1 / \rho_{1}^{2}(\mathbf{x}), 1 / \rho_{2}^{2}(\mathbf{x}), 0\right)$. Since the diagonal matrix has rank at most equal to two, we identify $\mathbf{u}_{3}$ with the outward unit normal $\mathbf{n}$ to $\Gamma$ at $\mathbf{x}$, coherently with the fact that metric $\mathcal{M}_{\Gamma}(\mathbf{x})$ is defined on the plane tangent to $\Gamma$ at $\mathbf{x}$. The definition of $\mathcal{M}_{\Gamma}$ may be particularized to a polyhedral surface $\Gamma_{h} \subset \mathbb{R}^{3}$, thus identifying the metric $\mathcal{M}_{\Gamma_{h}}$. In this context, it is standard to approximate $\mathcal{M}_{\Gamma_{h}}$ via a piecewise constant fuction on $\Gamma_{h}$.

Dealing with a mesh adaptation procedure, mesh $\Gamma_{h}$ becomes the unknown. In particular, evaluating the estimators $\eta_{I}, \eta_{G}$ on a background mesh, $\mathcal{T}_{h}^{B}$, we predict a metric $\mathcal{M}$, piecewise constant on $\mathcal{T}_{h}^{B}$, to generate a new adapted mesh, $\mathcal{T}_{h}^{A}$, which follows the directionalities of the function $f$ and of the surface $\Gamma$ with a desired accuracy. We define $\mathcal{M}$ such that $\left.\mathcal{M}\right|_{T}=\mathcal{U}_{T}^{\prime} \mathcal{R}_{T}^{-2} \mathcal{U}_{T}$, where, for any $T \in \mathcal{T}_{h}^{B}$, $\mathcal{U}_{T}=\left[\begin{array}{lll}\mathbf{u}_{1, T}^{*} & \mathbf{u}_{2, T}^{*} & \mathbf{0}\end{array}\right]^{\prime} \in \mathbb{R}^{3 \times 3}$ and $\mathcal{R}_{T}=\operatorname{diag}\left(\rho_{1, T}^{*}, \rho_{2, T}^{*}, 0\right) \in \mathbb{R}^{3 \times 3}$. Then, starting from the predicted metric $\mathcal{M}$, we employ a metric-based adaptive procedure to build the mesh $\mathcal{T}_{h}^{A}$. We have exploited the arbitrariness on $\mathbf{u}_{3, T}^{*}$ by identifying it with the vector identically equal to zero.

We aim at minimizing the number of elements to be employed for guaranteeing a given solution accuracy TOL by properly selecting the size, the shape, and the orientation of each element. In addition, we invoke a standard equidistribution criterion. We refer to the generic anisotropic error estimator

$$
\eta=\sum_{T \in \mathcal{T}_{h}} \eta_{T} \quad \text { with } \quad \eta_{T}=|T|^{3 / 2} \bar{\alpha}_{T} \bar{\nu}_{T}\left(\sigma_{T}, \mathbf{r}_{1, T}, g\right),
$$

where $\bar{\nu}_{T}\left(\sigma_{T}, \mathbf{r}_{1, T}, g\right)=\left(\sigma_{T} \mathbf{r}_{1, T}^{\prime} \bar{G}_{T}(g) \mathbf{r}_{1, T}+\frac{1}{\sigma_{T}} \mathbf{r}_{2, T}^{\prime} \bar{G}_{T}(g) \mathbf{r}_{2, T}\right)^{1 / 2}$, with $g$ a generic $H^{1}(\Gamma)$-function and $\bar{\alpha}_{T}=\alpha_{T} /|T|^{1 / 2}$ and $\bar{G}_{T}=G_{T} /|T|$ the dimensionless counterpart of $\alpha_{T}$ and of $G_{T}$, respectively. Estimator $\eta$ coincides with $\eta_{I}\left(g=f^{E}\right)$ or $\eta_{G}(g=d)$ or with a combination of them. Since both $\bar{\alpha}_{T}$ and $\bar{\nu}_{T}$ are dimensionless, the information related to the area of $T$ in (4.1) is essentially lumped in the factor $|T|^{3 / 2}$ (at least asymptotically). According to a predictive approach, we compute the quantities $\bar{\alpha}_{T}$, $|T|=|\widehat{T}| s_{1, T} s_{2, T}, \bar{G}_{T}(g)$ on the background grid, while $\sigma_{T}$ and $\mathbf{r}_{1, T}$ become the actual unknowns. By extending the approach proposed, e.g., in [29], to a surface setting, we are led to solve, for each element $T \in \mathcal{T}_{h}^{B}$, the local constrained minimization problem:

find $\sigma_{T}^{*}, \mathbf{r}_{1, T}^{*}$ s.t. $\bar{\nu}_{T}\left(\sigma_{T}^{*}, \mathbf{r}_{1, T}^{*}, g\right)$ is minimized, with $\sigma_{T}^{*} \geq 1, \mathbf{r}_{i, T}^{*} \cdot \mathbf{r}_{j, T}^{*}=\delta_{i j}, i, j=1,2$.

This problem guarantees the minimization of the cardinality of the adapted mesh $\mathcal{T}_{h}^{A}$ and does not demand any extra computational burden since it is explicitly solvable. Successively, by combining (4.2) with the equidistribution of the error, we derive the optimal metric via the elemental matrices $\left.\mathcal{M}\right|_{T}=\mathcal{U}_{T}^{\prime} \mathcal{R}_{T}^{-2} \mathcal{U}_{T}$ with

$$
\mathcal{U}_{T}=\left[\begin{array}{lll}
\mathbf{u}_{1, T}^{*} & \mathbf{u}_{2, T}^{*} & \mathbf{0}
\end{array}\right]^{\prime}, \quad \mathcal{R}_{T}=\operatorname{diag}\left(\rho_{1, T}^{*}, \rho_{2, T}^{*}, 0\right),
$$

where $\mathbf{u}_{1, T}^{*}=\mathbf{w}_{2, T}, \mathbf{u}_{2, T}^{*}=\mathbf{w}_{1, T}$,

$$
\rho_{1, T}^{*}=\left(\frac{1}{2^{1 / 2}}\left(\frac{\mu_{1, T}}{\mu_{2, T}^{2}}\right)^{1 / 2} \frac{\text { TOL }}{\# \mathcal{T}_{h}^{B} \bar{\alpha}_{T}|\widehat{T}|^{3 / 2}}\right)^{1 / 3},
$$

Copyright (c) by SIAM. Unauthorized reproduction of this article is prohibited. 

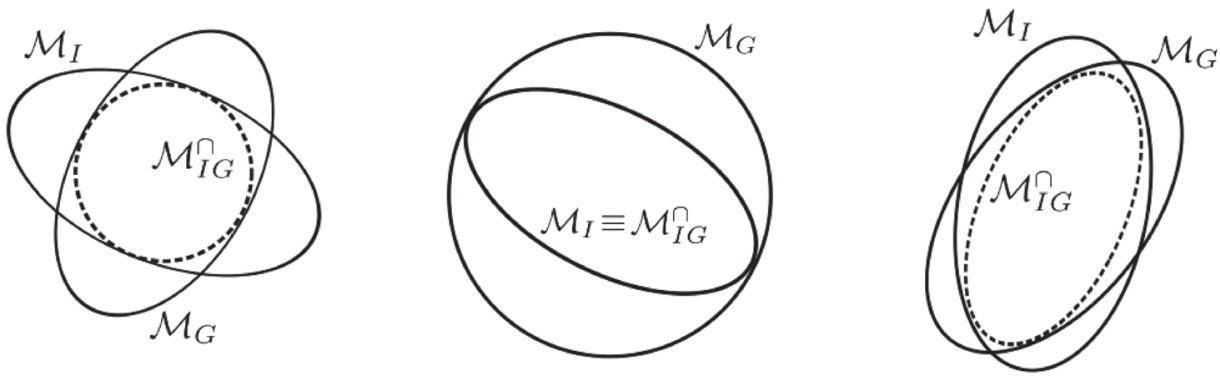

FIG. 2. Examples of metric intersection $\mathcal{M}_{I} \cap \mathcal{M}_{G}$.

$$
\rho_{2, T}^{*}=\left(\frac{1}{2^{1 / 2}}\left(\frac{\mu_{2, T}}{\mu_{1, T}^{2}}\right)^{1 / 2} \frac{\mathrm{TOL}}{\# \mathcal{T}_{h}^{B} \bar{\alpha}_{T}|\widehat{T}|^{3 / 2}}\right)^{1 / 3},
$$

with $\left(\mu_{i, T}, \mathbf{w}_{i, T}\right)$, for $i=1,2$, the eigenvalue-eigenvector pairs of the matrix $\bar{G}_{T}(g)$, with $\mu_{1, T} \geq \mu_{2, T}$. Finally, metric $\mathcal{M}$ is employed to generate the adapted mesh $\mathcal{T}_{h}^{A}$ via the procedure detailed in section 5.1.

4.2. Combination of metrics. We consider the issue of simultaneously dealing with the metrics $\mathcal{M}_{I}$ and $\mathcal{M}_{G}$ associated with estimator $\eta_{I}$ and $\eta_{G}$, respectively. Both these metrics are computed starting from the same background grid $\mathcal{T}_{h}^{B}$, i.e., for each element $T \in \mathcal{T}_{h}^{B}$, we have two predictions for the optimal size, shape, and orientation. The role played by $\mathcal{M}_{I}$ and $\mathcal{M}_{G}$ is different: $\mathcal{M}_{I}$ is instrumental to controlling the error related to the function $f$, while $\mathcal{M}_{G}$ is meant to limit the error due to the approximation of $\Gamma$ via $\Gamma_{h}$. The idea is to combine $\mathcal{M}_{I}$ and $\mathcal{M}_{G}$ to merge both benefits. For this purpose, we investigate three techniques, so that the metric $\mathcal{M}_{I G}$ driving the adaptation coincides with the following:

(a) The metric intersection $\mathcal{M}_{I G}^{\cap} \equiv \mathcal{M}_{I} \cap \mathcal{M}_{G}$, based on the simultaneous reduction of the metrics $\mathcal{M}_{I}$ and $\mathcal{M}_{G}$ (we refer to Chapter 10 in [20] for the technical details). This approach represents the most straightforward way to merge the discretization with the geometric error control. Nevertheless, in some circumstances, the results are overly conservative since the intersection of two anisotropic metrics does not necessarily yield an anisotropic metric (see Figure 2, left, for an example).

(b) The maximum metric $\mathcal{M}_{I G}^{\max }$, such that $\left.\mathcal{M}_{I G}^{\max }\right|_{T}$ is the metric associated with the maximum local estimator between $\eta_{I, T}$ and $\eta_{G, T}$, for any $T \in \mathcal{T}_{h}^{B}$.

(c) A convex combination of the two metrics $\mathcal{M}_{I}$ and $\mathcal{M}_{G}$. In particular, we start from the convex combination $\gamma \eta_{I, T}+(1-\gamma) \eta_{G, T}$ of the local estimators, with $\gamma \in[0,1]$. Then, following Proposition 5.4 in [29], we advantageously exploit the common structure shared by $\eta_{I, T}$ and $\eta_{G, T}$ to combine the two metrics into the single estimator $\eta_{I G, T}^{\gamma}=|T|^{3 / 2} \bar{\alpha}_{T} \bar{\nu}_{T}^{\gamma}\left(\sigma_{T}, \mathbf{r}_{1, T}, f^{E}, d\right)$, where

$$
\bar{\nu}_{T}^{\gamma}\left(\sigma_{T}, \mathbf{r}_{1, T}, f^{E}, d\right)=\left(\sigma_{T} \mathbf{r}_{1, T}^{\prime} \bar{G}_{T}^{\gamma}\left(f^{E}, d\right) \mathbf{r}_{1, T}+\frac{1}{\sigma_{T}} \mathbf{r}_{2, T}^{\prime} \bar{G}_{T}^{\gamma}\left(f^{E}, d\right) \mathbf{r}_{2, T}\right)^{1 / 2}
$$

with $\bar{G}_{T}^{\gamma}\left(f^{E}, d\right)=\gamma^{2} \bar{G}_{T}\left(f^{E}\right)+(1-\gamma)^{2} \bar{G}_{T}(d)$. By mimicking the procedure employed to convert estimator (4.1) into the optimal metric in (4.3), we get the metric $\mathcal{M}_{I G}^{\gamma}$, automatically blending the interpolation with the geometric information. 

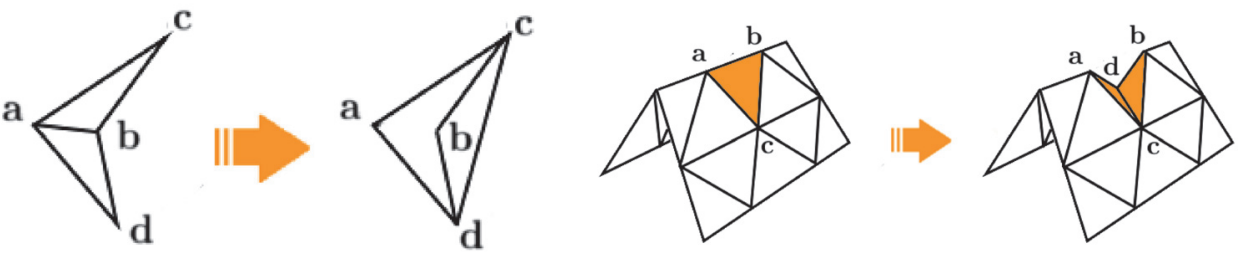

FIG. 3. Instances of an unswappable edge (ab) for a planar (left) and a nonplanar (right) mesh.

Approach (c) allows us to skip the computation of the distinct optimal metrics $\mathcal{M}_{I}$ and $\mathcal{M}_{G}$. A single optimal metric is derived after defining the new error estimator $\eta_{I G}^{\gamma}=\sum_{T \in \mathcal{T}_{h}^{B}} \eta_{I G, T}^{\gamma}$

5. Numerical assessment. After setting the adaptive procedure to commute $\mathcal{M}_{I G}$ into practical operations yielding the adapted mesh $\mathcal{T}_{h}^{A}$, we assess the robustness of the proposed adaptive tool with two test cases.

5.1. The adaptive procedure. The adapted mesh is generated via a metricbased procedure. This represents a standard approach in an anisotropic framework (see, e.g., $[19,10,5,27])$. The goal is to obtain an optimal mesh $\mathcal{T}_{h}^{A}$ such that each element $T \in \mathcal{T}_{h}^{A}$ coincides with a unitary equilateral triangle with respect to the metric $\mathcal{M}_{I G}$, i.e., $\|e\|_{\mathcal{M}_{I G}}=\sqrt{e^{\prime} \mathcal{M}_{I G} e}=1$, for any $e \in T$ and for any $T \in \mathcal{T}_{h}^{A}$. Since this target is not exactly reachable in a general case, we devise an optimization procedure to minimize the distance of the adapted grid to the optimal one, in a sense that is described in the following. We resort to the following multichoice criterion: for any edge $e$ of the skeleton $\mathcal{E}_{B}$ of the background grid,

(i) if $\|e\|_{\mathcal{M}_{I G}} \simeq 1$, the edge $e$ already has the optimal length with respect to the predicted metric and can be directly identified with an edge of the adapted $\operatorname{mesh} \mathcal{T}_{h}^{A}$

(ii) if $\|e\|_{\mathcal{M}_{I G}} \gg 1$, the edge $e$ is too long according to the predicted metric;

(iii) if $\|e\|_{\mathcal{M}_{I G}} \ll 1$, the edge $e$ is too short according to the predicted metric.

In both the cases (ii) and (iii), the edge $e$ has to be properly modified before being included in $\mathcal{T}_{h}^{A}$. For this purpose, we employ an iterative procedure based on local operations. The meshes playing the role of background and adapted grid are updated during the iterative process. To compute the length $\|e\|_{\mathcal{M}_{I G}}$ starting from the elemental metric $\left.\mathcal{M}_{I G}\right|_{T}$, we first assign a metric $\mathcal{M}_{I G}^{\mathbf{z}}$ to each node $\mathbf{z}$ of the current mesh by computing the mean of the metrics associated with the triangles of the patch $\omega_{\mathbf{z}}$. Then, let $\mathbf{a}$ and $\mathbf{b}$ be the endpoints of the edge $e$ and let $\mathcal{M}_{I G}^{\mathbf{a}}$ and $\mathcal{M}_{I G}^{\mathbf{b}}$ be the corresponding metrics. We compute $\|e\|_{\mathcal{M}_{I G}}=\max \left(\|e\|_{\mathcal{M}_{I G}^{\mathrm{a}}},\|e\|_{\mathcal{M}_{I G}^{\mathrm{b}}}\right)$, where $\|e\|_{\mathcal{M}_{I G}^{z}}=\sqrt{e^{\prime} \mathcal{M}_{I G}^{\mathbf{z}} e}$, for $\mathbf{z}=\mathbf{a}, \mathbf{b}$.

5.1.1. Local operations. We use four different operations.

A. Edge swapping. Edge swapping turns out to be among the most efficient and effective local operations to anisotropically modify a generic triangular mesh $[18,6]$. It is not always possible to apply edge swapping even in a planar framework. With reference to Figure 3, we can swap the edge ab if the following conditions are verified:

(R1) The edge cd does exist in the mesh (the meaning of this condition is clarified in the next section).

(R2) There does not exist an obtuse angle adjacent to the edge ab. Figure 3, left, furnishes an example of an unswappable edge. The new edge cd leads 
to occupying a portion of the domain not included before in $\Delta_{\text {abc }} \cup \Delta_{\text {bad }}$. Moreover, the conformity of the mesh is compromised.

The edge swapping becomes a more complex operation when dealing with a triangular surface mesh, due to the intrinsic curvature of the surface. This is particularly troublesome where the mesh exhibits ridges. As exemplified in Figure 3, right, edge swapping may lead to an incorrect approximation of the surface by violating the corresponding curvature. To overcome this issue, we check an additional condition:

(R3) The angle $\theta$ between the normals to the faces $\Delta_{\text {abc }}$ and $\Delta_{\text {bad }}$ is smaller than a minimum threshold $\theta_{\min }$ (in the numerical validation below, we set $\theta_{\min }=15^{\circ}$.

After verifying the geometric and topological consistency of the edge swapping, we fix the following criterion in view of the mesh adaptation: we swap the edge $\mathbf{a b}$ if

$$
\left.|||\mathbf{c d}|\right|_{\mathcal{M}_{I G}}-1|<|\|\mathbf{a b}\|_{\mathcal{M}_{I G}}-1 \mid \text {. }
$$

We settle a new edge swapping routine starting from the well-known Lawson flip algorithm for the construction of a 2D planar Delaunay triangulation [28]. In particular, we modify the original algorithm to deal with surface meshes and to include the metric-based check (5.1). The algorithm may be applied to all the edges of the grid or just to a subset of elements. For the sake of simplicity, we assume that, at the beginning, $S$ contains only the edge ab. Then, the algorithm reads as follows.

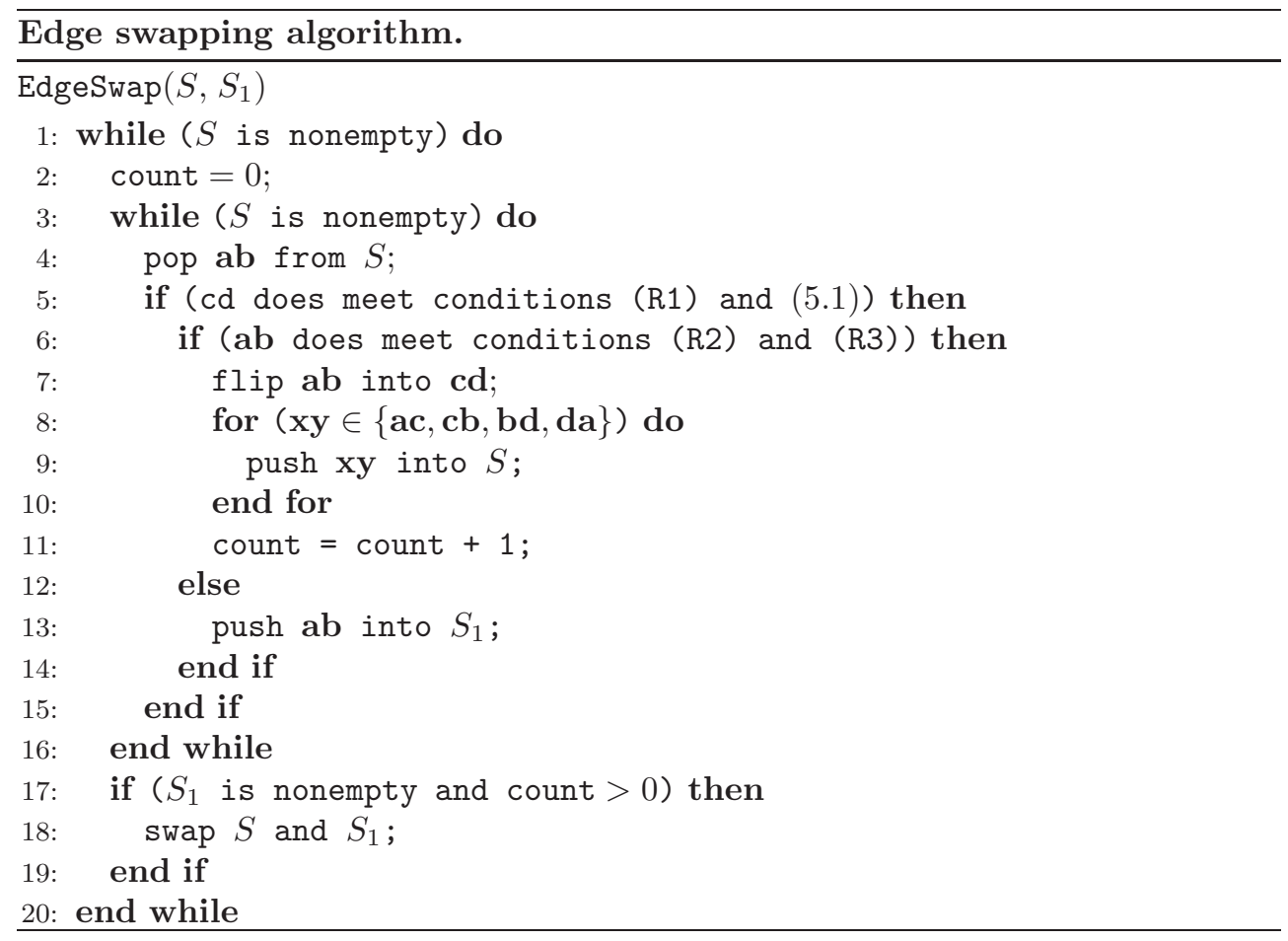

In the inner loop (lines 3-16), according to the Lawson procedure, the check for the swapping propagates from the edges contained in $S$ and recognized as swappable to the corresponding neighboring edges. The three topological criteria (R1)-(R3) and the metric check (5.1) have to be satisfied to consider an edge as swappable. In particular, if the edge ab does not meet criteria (R2) and (R3) even though the edge cd satisfies conditions (R1) and (5.1), the edge $\mathbf{a b}$ is automatically moved to stack $S_{1}$. 
Once an edge has been swapped, it will never be regenerated. Nevertheless, before closing the procedure, the external loop (lines 17-19) performs an additional check on the edges previously discarded since identifying a nonvalid topological configuration even though leading to an improvement in terms of metric.

B. Edge splitting. This operation plays an crucial role in view of local refinements [34]. The idea is to increase the mesh resolution where the solution exhibits strong variations, by locally reducing the size of the mesh and by stretching the elements according to the directional features of the solution. The edges to be split, i.e., with a length predicted by $\mathcal{M}_{I G}$ greater than one, are halved by inserting a new vertex at the corresponding midpoint. Extra care has to be taken since we are dealing with a polyhedral surface. As depicted in Figure 4, middle, the addition of the new vertex $\mathbf{v}$ does not necessarily lead to an improvement in fitting $\Gamma$ via $\Gamma_{h}$. As a consequence, after the addition of the new vertex, we project $\mathbf{v}$ on the surface $\Gamma$ by resorting to the algorithm proposed in [24]. This simple device necessarily yields an effective improvement in the approximation of $\Gamma$ as shown in Figure 4, right.

C. Edge collapsing. The most significative operation in view of a mesh coarsening is the edge collapsing. To this aim, we resort to an edge contraction technique (e.g., $[26,11,32])$, after identifying the edges $e$ with $\|e\|_{\mathcal{M}_{I G}} \ll 1$. The endpoints of $e$ are moved toward each other until they both coincide with a new vertex v. Different choices are possible to fix vertex $\mathbf{v}$. We usually select the midpoint of the edge to be contracted unless this choice leads to invalid topological configurations, e.g., to inverted triangles (see Figure 5, left). Following [14], to overcome this issue we make an additional check on the sets of vertices connected to $\mathbf{a}$ and $\mathbf{b}$, respectively. In particular, if the intersection between these two sets includes any point different from the vertices of the faces $\Delta_{\text {bax }}$ and $\Delta_{\text {bay }}$, the contraction of the edge ab into the vertex $\mathbf{v}$ leads to an inverted triangle. With reference to Figure 5, left, the presence of the point $\mathbf{c}$ justifies the failure of the contraction algorithm. Finally, exactly as for the edge splitting, after any edge contraction we have to project the new position of the vertex $\mathbf{v}$ on the surface $\Gamma$ to actually guarantee the fitting of the surface at hand.

D. Node smoothing. Node smoothing improves the quality of a mesh. In contrast to the previous ones, this operation simply moves the nodes of the mesh into new positions without modifying the mesh topology. We may provide a physical interpretation of this operation by identifying the patch $\Delta_{\mathbf{V}}$ of elements associated with the vertex $\mathbf{v}$ with a system of springs. Thus, the smoothing procedure aims at locating the vertex $\mathbf{v}$ in the position that minimizes the elastic energy of the whole system. In an isotropic context, the smoothing moves $\mathbf{v}$ to the barycenter of $\Delta_{\mathbf{v}}$. On the contrary, when dealing with an anisotropic mesh adaptation, we have to properly include the effect of the metric, for instance, by varying the stiffness coefficient of the different springs. Exactly as for the edge collapsing, the new position for the point $\mathbf{v}$ may lead to invalid configurations (see Figure 5, right) where inverted elements are generated. As a consequence, a careful check on the predicted new configuration is performed before applying any smoothing.

5.1.2. The adaptation sequence. The iterative procedure leading to the optimal mesh with $\|e\|_{\mathcal{M}_{I G}} \approx 1$ might be strongly affected by the sequence of local operations applied to the initial mesh. We resort to the following strategy. The edge splitting and the edge collapsing, in combination with a local edge swapping and followed by a global edge swapping, are iteratively repeated, together with a cycle of runs of node smoothing. 

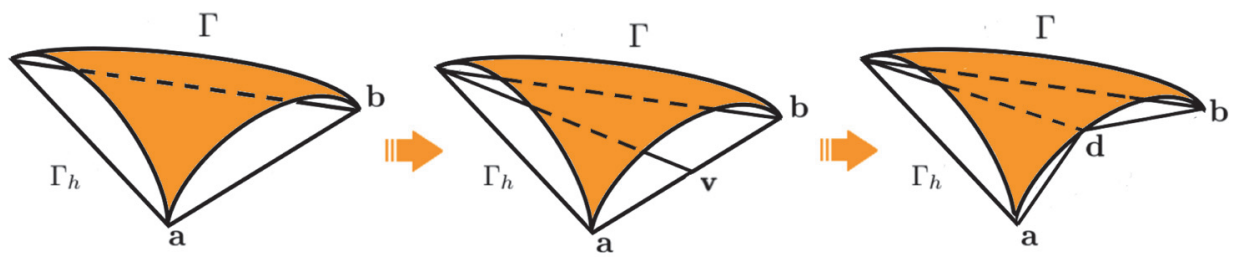

FIG. 4. Splitting of the edge $\mathbf{a b}$ via the addition of the point $\mathbf{v}$ and projection of $\mathbf{v}$ on the surface $\Gamma$ via point $\mathbf{d}$.
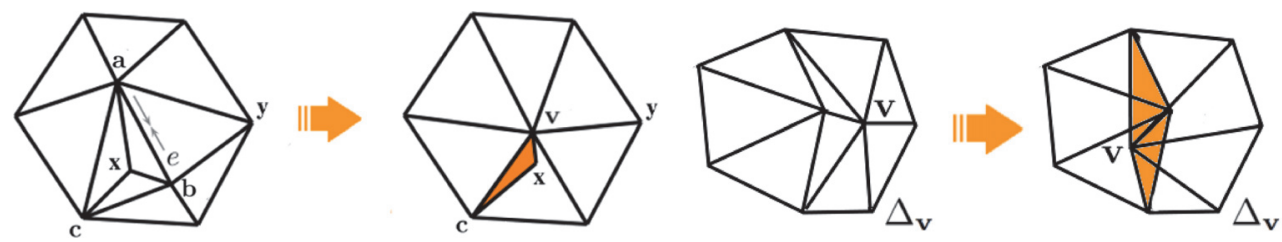

FIG. 5. Invalid topological configuration yielded by the contraction of the edge (left) and by the smoothing of vertex $\mathbf{v}$ (right).

The iterative procedure is controlled by three different flags, involving the tolerance TOL on the approximation accuracy, a maximum number maxIter of adaptive iterations, and a check on the cardinality of the mesh:

(FL1) errCheck: If the global error evaluated on the adapted mesh via $\eta_{I G}=$ $\eta_{I}+\eta_{G}$ is greater than TOL, errCheck is set true; otherwise it is false.

(FL2) iterCheck: The value of this flag is true until the number of iterations is lower than maxiter.

(FL3) meshCheck: We compute $\# \mathcal{T}_{\text {diff }}=\left|\# \mathcal{T}_{\text {old }}-\# \mathcal{T}_{\text {new }}\right| / \# \mathcal{T}_{\text {old }}$, with $\# \mathcal{T}_{\text {old }}$ and $\# \mathcal{T}_{\text {new }}$ the cardinality of the mesh before and after the adaptation, respectively. Then, if $\# \mathcal{T}_{\text {diff }}>0.05$, meshCheck is true; otherwise it is false.

The whole adaptive algorithm reads as follows.

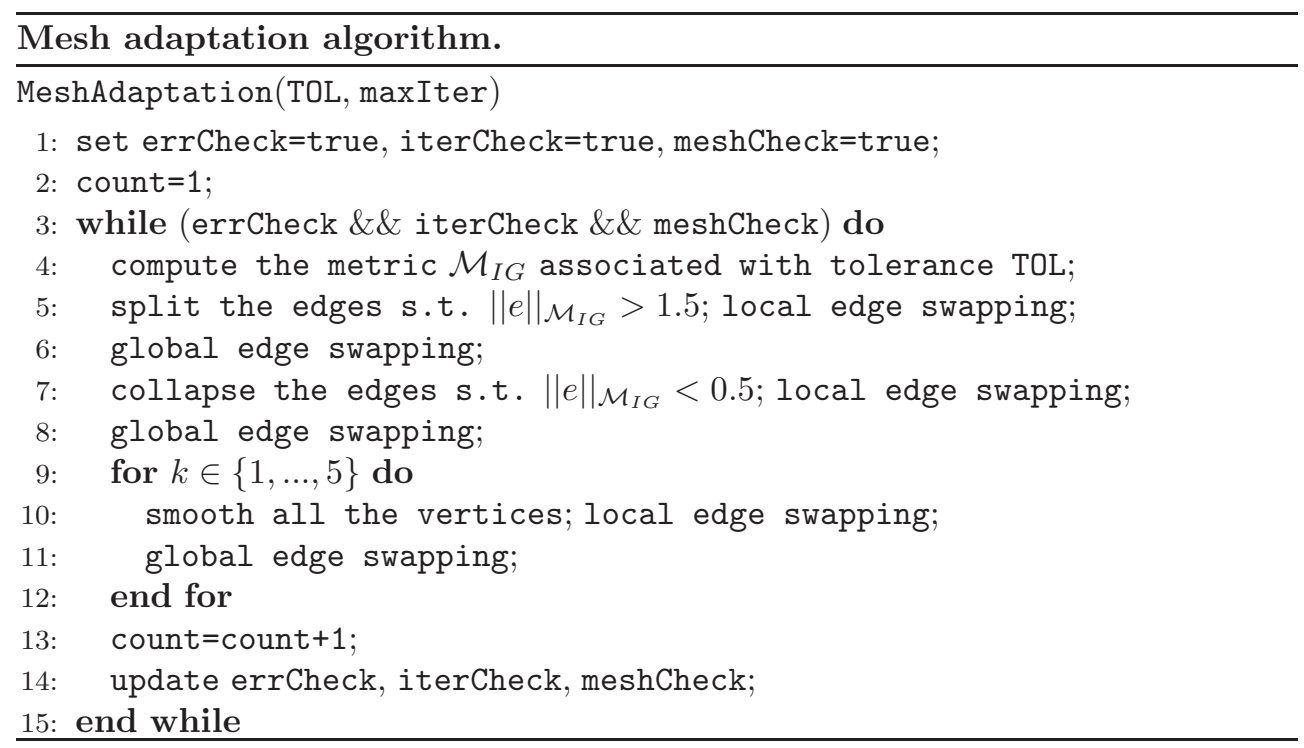

Copyright (c) by SIAM. Unauthorized reproduction of this article is prohibited. 
The adaptive procedure stops when one of the flags (FL1)-(FL3) is false. We highlight the intensive employment of the function EdgeSwap, both at a local (lines 5, 7,10 ) and at a global (lines $6,8,11$ ) level, in accordance with the goal of generating an anisotropic mesh. Finally, the number of node smoothing iterations is heuristically set.

The meaning of request (R1) becomes clear in light of the whole adaptive procedure. Since the swapping quickly propagates to the edges of the mesh, an edge marked as swappable might be previously involved by other operations before swappig actually takes place.

5.2. Test cases. We investigate the robustness of the error analysis as well as of the adaptive procedure on both an open and a closed surface. Starting from the same grid, we compare the adapted meshes generated via four different metrics, namely, $\mathcal{M}_{I G}^{\cap}, \mathcal{M}_{I G}^{\max }, \mathcal{M}_{I G}^{\gamma}$ defined in section 4.2 together with the metric $\mathcal{M}_{I}$ taking into account the interpolation error only. A quantitative investigation of the anisotropic analysis is also provided.

5.2.1. Test case 1: An open surface. Let $\Gamma_{1}$ be the sinusoidal surface defined by $d_{1}:[0,1] \times[0,1] \times[-0.2,0.2] \rightarrow \mathbb{R}$ with $d_{1}(\mathbf{x})=0.2 \cos \left(\pi x_{1}\right) \cos \left(\pi x_{2}\right)-x_{3}$. We consider the function $f_{1}: \Gamma_{1} \rightarrow \mathbb{R}$ with $f_{1}(\mathbf{x})=4 x_{2}\left(1-x_{1}\right)\left(1-x_{2}\right)\left(1-e^{-1000 x_{1}}\right)$. Figure 6 , left, depicts the colorplot of $f_{1}$ on $\Gamma_{1}$. The solution exhibits a boundary layer along $\left\{\left(0, x_{2}\right), 0 \leq x_{2} \leq 1\right\}$, where it reaches its maximum value. We run the adaptive procedure by making different choices for $\mathcal{M}_{I G}$. In particular, in algorithm MeshAdaptation, we set TOL $=6$. e- 05 , maxIter $=10$, while we choose the value $2 / 3$ for the parameter $\gamma$ combining the interpolation with the geometric metrics.

Comparison among metrics. Figure 7 compares a detail of the adapted grid associated with the metric $\mathcal{M}_{I G}^{\Upsilon}, \mathcal{M}_{I G}^{\max }, \mathcal{M}_{I G}^{\gamma}$, and $\mathcal{M}_{I}$ for a similar number (about 8700 ) of elements. In particular, we focus on the boundary layer. It is correctly detected by all the metrics and the elements are properly stretched. Nevertheless, the anisotropic features of the mesh are significantly less evident when the metric intersection drives the adaptive procedure (compare, e.g., Figure 7(a) with Figure 7(b)). This is confirmed by the maximum value $\sigma_{\max }=\max _{T \in \mathcal{T}_{h}^{A}} \sigma_{T}$ of the corresponding aspect ratio, as shown in Table 1, left panel. Moreover, the mesh in Figure 7(d) obtained via $\mathcal{M}_{I}$ exhibits elements correctly stretched as well, with a maximum aspect ratio similar to the one associated with $\mathcal{M}_{I G}^{\max }$.

The benifits led by the geometric information are evident if we drastically diminish the number of elements (i.e., if we increase the value of TOL). As Figure 8(d) shows, the quality of the approximation provided by $\mathcal{M}_{I}$ becomes very poor in such a case. The four adapted meshes are generated after fixing TOL $=8 \mathrm{e}-04$ and are characterized
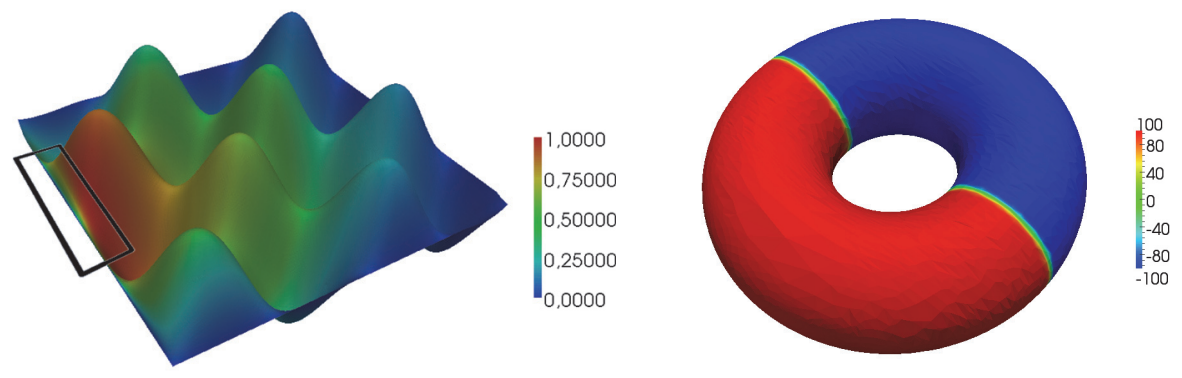

FIG. 6. Function $f_{1}$ on the surface $\Gamma_{1}$ (left); function $f_{2}$ on the surface $\Gamma_{2}$ (right). 


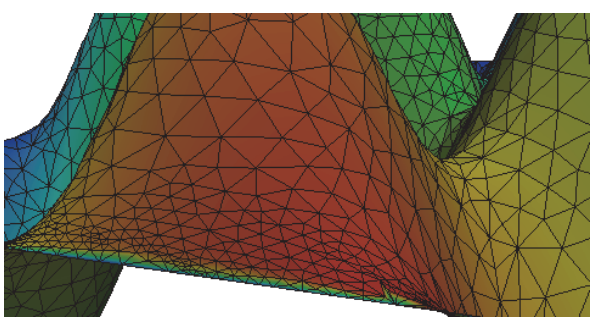

(a)

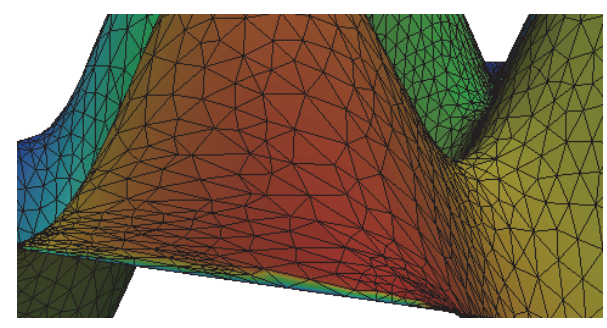

(c)

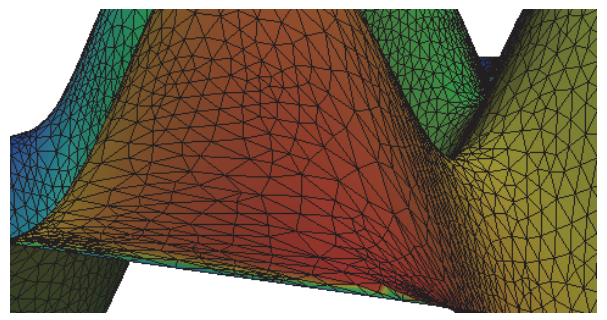

(b)

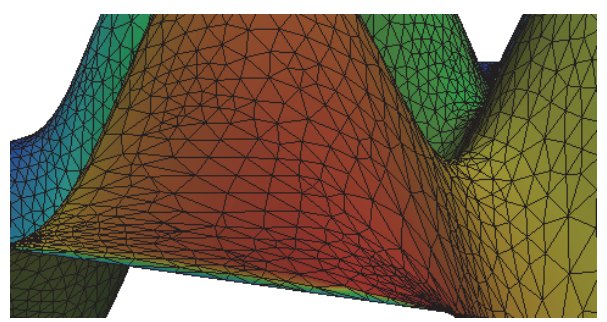

(d)

FIG. 7. Test case 1: detail in correspondence with the boundary layer of the adapted mesh $\mathcal{T}_{h}^{A}$ generated via the metric (a) $\mathcal{M}_{I G}^{\cap}$, (b) $\mathcal{M}_{I G}^{\max }$, (c) $\mathcal{M}_{I G}^{\gamma}$, and (d) $\mathcal{M}_{I}$.

TABLE 1

Maximum aspect ratio and surface mismatch for test case 1 (left panel) and for test case 2 (right panel).

\begin{tabular}{|c|c|c||c|c|}
\hline & \multicolumn{2}{|c||}{ Test Case 1 } & \multicolumn{2}{c|}{ Test Case 2} \\
\hline Metric & $\sigma_{\max }$ & $d_{\max }$ & $\sigma_{\max }$ & $d_{\max }$ \\
\hline $\mathcal{M}_{I G}^{\cap}$ & 4.93 & $7.923 \mathrm{e}-03$ & $9.967 \mathrm{e}+00$ & $2.174 \mathrm{e}-02$ \\
\hline $\mathcal{M}_{I G}^{\max }$ & 28.05 & $7.785 \mathrm{e}-03$ & $3.105 \mathrm{e}+01$ & $2.359 \mathrm{e}-02$ \\
\hline $\mathcal{M}_{I G}^{\gamma}$ & 36.97 & $8.537 \mathrm{e}-03$ & $2.601 \mathrm{e}+01$ & $2.259 \mathrm{e}-02$ \\
\hline $\mathcal{M}_{I}$ & 22.68 & $8.901 \mathrm{e}-02$ & $6.176 \mathrm{e}+01$ & $1.168 \mathrm{e}-01$ \\
\hline
\end{tabular}

by about 2600 elements. Metric $\mathcal{M}_{I}$ does not correctly describe surface $\Gamma_{1}$ with an evident loss of accuracy, for instance, in detecting the peak at the corner $(1,1)$. On the contrary, despite the limited number of elements, the geometric information integrated in $\mathcal{M}_{I G}^{\cap}, \mathcal{M}_{I G}^{\max }, \mathcal{M}_{I G}^{\gamma}$ is enough to meet the main geometric features of $\Gamma_{1}$. The best approximation in terms of geometry fitting is provided by $\mathcal{M}_{I G}^{\max }$. This is confirmed by the values of $d_{\max }=\max _{K \in \mathcal{T}_{h}^{A}}\left\|p_{K}-b_{K}\right\|_{2}$ in the left panel of Table 1 , which measures the mismatch between $\Gamma_{1}$ and $\mathcal{T}_{h}^{A}$, for the four meshes in Figure 8 , $b_{K}$ being the barycenter of the triangle $K$ and $p_{K}$ the corresponding projection on the surface $\Gamma_{1}$, with $\|\cdot\|_{2}$ the standard Euclidean norm. As expected the largest value of $d_{\max }$ is associated with the mesh predicted by $\mathcal{M}_{I}$.

Anisotropy versus isotropy. The computational advantages led by an anisotropic versus an isotropic mesh adaptation are well-established in the literature (see, e.g., $[7,8,29,36])$. To make this analysis quantitative, we introduce the following definitions:

$$
e_{\mathrm{tot}}=\left\|f-I_{h} f^{E}\right\|_{L^{1}(\Gamma)}, \quad e_{\text {mean }}=\frac{e_{\text {tot }}}{\sum_{T \in \mathcal{T}_{h}^{A}}|T|}, \quad e_{\max }=\max _{T \in \mathcal{T}_{h}^{A}}\left\|f-I_{h} f^{E}\right\|_{L^{1}(T)}^{*} .
$$




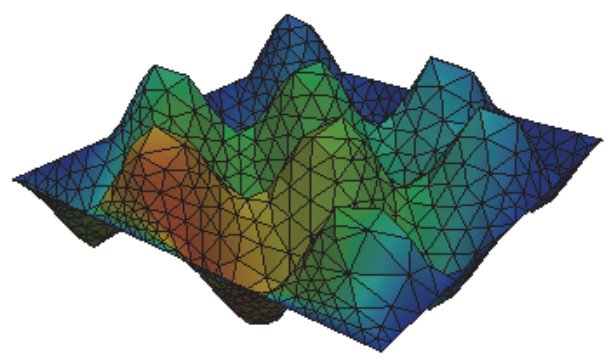

(a)

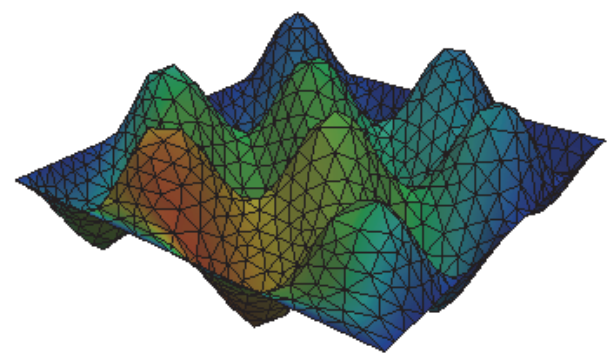

(c)

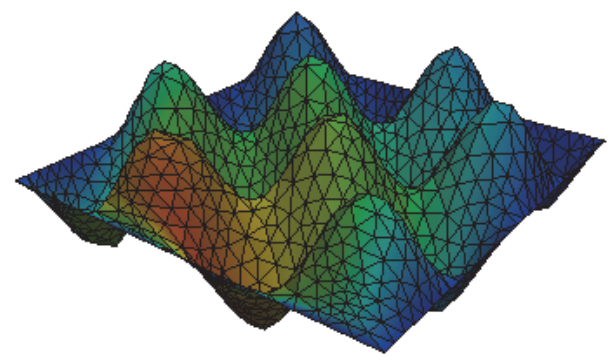

(b)

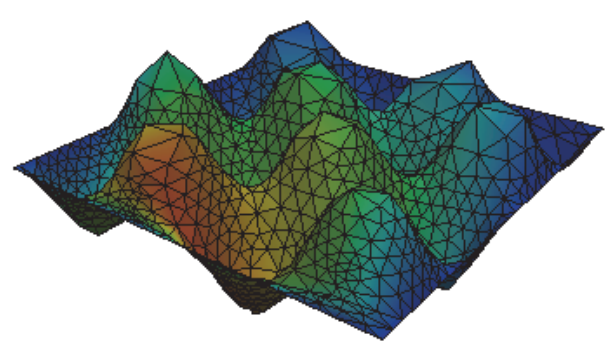

(d)

FIG. 8. Test case 1: adapted mesh $\mathcal{T}_{h}^{A}$ generated via the metric (a) $\mathcal{M}_{I G}^{\cap}$, (b) $\mathcal{M}_{I G}^{\max }$, (c) $\mathcal{M}_{I G}^{\gamma}$ and $(\mathrm{d}) \mathcal{M}_{I}$

TABLE 2

Test case 1: anisotropy versus isotropy for a fixed cardinality of the mesh.

\begin{tabular}{c|cc|cc|cc}
\hline & \multicolumn{2}{|c|}{$\mathcal{M}_{I}$} & \multicolumn{2}{c|}{$\mathcal{M}_{I G}^{\max }$} & \multicolumn{2}{c}{$\mathcal{M}_{I G}^{\gamma}$} \\
\hline & Isotropy & Anisotropy & Isotropy & Anisotropy & Isotropy & Anisotropy \\
\hline Elements & 2664 & 2618 & 3483 & 3434 & 2952 & 2925 \\
\hline$e_{\text {tot }}$ & $3.654 \mathrm{e}-03$ & $1.195 \mathrm{e}-03$ & $3.648 \mathrm{e}-03$ & $9.160 \mathrm{e}-04$ & $4.334 \mathrm{e}-03$ & $1.392 \mathrm{e}-03$ \\
$e_{\text {mean }}$ & $1.371 \mathrm{e}-06$ & $4.568 \mathrm{e}-07$ & $1.047 \mathrm{e}-06$ & $2.667 \mathrm{e}-07$ & $1.468 \mathrm{e}-06$ & $4.762 \mathrm{e}-07$ \\
$e_{\max }$ & $6.431 \mathrm{e}-05$ & $3.135 \mathrm{e}-05$ & $6.566 \mathrm{e}-05$ & $1.669 \mathrm{e}-05$ & $1.027 \mathrm{e}-04$ & $5.424 \mathrm{e}-05$ \\
\hline
\end{tabular}

In particular, to compute $e_{\text {tot }}$, we employ the equality in the proof of Proposition 3.2,

$$
\left\|f-I_{h} f^{E}\right\|_{L^{1}(\Gamma)}=\int_{\Gamma_{h}}\left|f^{E}(\mathbf{x})-I_{h} f^{E}(\mathbf{x})\right|\left|\mu_{h}(\mathbf{x})\right| d \Gamma_{h},
$$

whereas $\| f-\left.I_{h} f^{E}\right|_{L^{1}(T)} ^{*}=\int_{T}\left|f^{E}(\mathbf{x})-I_{h} f^{E}(\mathbf{x})\right|\left|\mu_{h}(\mathbf{x})\right| d T$ is derived by localizing (5.3) to face $T$. First, we verify that anisotropy provides an improvement in terms of accuracy for a fixed cardinality of the adapted mesh. Table 2 exemplifies such a trend. We have excluded from this comparison the adaptive procedure based on $\mathcal{M}_{I G}^{\cap}$ due to the limited anisotropy characterizing such an approach. While a not so striking difference on the maximum error is obtained, we appreciate a reduction of at least one-third or more on the total error as well as a gain of one order on the mean error. Then, we perform the dual check, i.e., we fix the accuracy TOL and we assess the gain in terms of computational cost. The results are collected in Table 3. The dimension of the linear system we are led to solve in the anisotropic framework is remarkably lower. The maximum gain is yielded by the approach based on the maximum metric (one-ninth of elements!) and, also in the worst case, i.e., for $\mathcal{M}_{I}$, we reduce of one-fifth the number of degrees of freedom. 
TABLE 3

Test case 1: anisotropy versus isotropy for a fixed accuracy of the mesh.

\begin{tabular}{c|cc|cc|cc}
\hline & \multicolumn{2}{|c|}{$\mathcal{M}_{I}$} & \multicolumn{2}{c|}{$\mathcal{M}_{I G}^{\max }$} & \multicolumn{2}{c}{$\mathcal{M}_{I G}^{\gamma}$} \\
\hline & Isotropy & Anisotropy & Isotropy & Anisotropy & Isotropy & Anisotropy \\
\hline Elements & 11479 & 2009 & 18201 & 2024 & 16548 & 2031 \\
\hline$e_{\text {tot }}$ & $3.168 \mathrm{e}-03$ & $3.529 \mathrm{e}-03$ & $3.159 \mathrm{e}-03$ & $3.373 \mathrm{e}-03$ & $3.049 \mathrm{e}-03$ & $3.441 \mathrm{e}-03$ \\
$e_{\text {mean }}$ & $2.759 \mathrm{e}-07$ & $1.756 \mathrm{e}-06$ & $1.735 \mathrm{e}-07$ & $1.666 \mathrm{e}-06$ & $1.842 \mathrm{e}-07$ & $1.694 \mathrm{e}-06$ \\
$e_{\max }$ & $6.557 \mathrm{e}-05$ & $1.814 \mathrm{e}-04$ & $6.619 \mathrm{e}-05$ & $2.089 \mathrm{e}-04$ & $7.004 \mathrm{e}-05$ & $1.651 \mathrm{e}-04$ \\
\hline
\end{tabular}
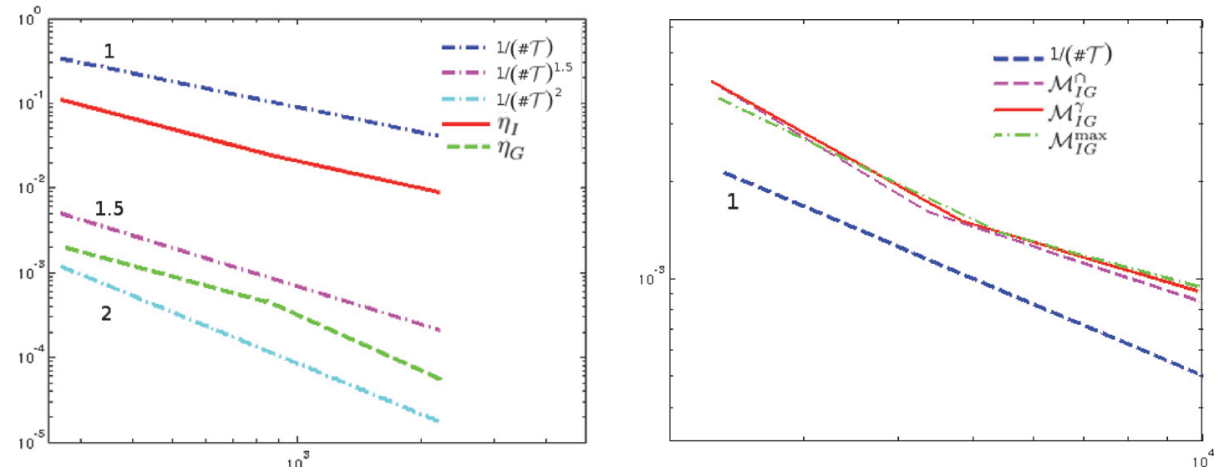

FIG. 9. Test case 1: convergence history for the estimators $\eta_{I}$ (solid line) and $\eta_{G}$ (dashed line) in a loglog plot (left); convergence trend of the global error estimator for three different metrics in a loglog plot (right).

A comparison of Tables 2 and 3 does not identify a most strategical approach in terms of computational advantages provided by the anisotropic adaptation. Indeed, by properly scaling the error with respect to the number of elements, we obtain a similar computational gain for $\mathcal{M}_{I}, \mathcal{M}_{I G}^{\max }, \mathcal{M}_{I G}^{\gamma}$.

Convergence analysis. We deal with a twofold check. First, we perform an asymptotic analysis of both the interpolation and the geometric error estimators. As shown in Figure 9, left, the estimator $\eta_{I}$ exhibits the expected trend as a function of the mesh cardinality $\# \mathcal{T}$, namely, the rate of convergence turns out to be of the first order with respect to $1 / \# \mathcal{T}$. Concerning the geometric error estimator, according to [12], we expect a higher order of convergence. This statement is confirmed by the trend of $\eta_{G}$ characterized by an order of about 1.5. The different order of convergence of $\eta_{I}$ and $\eta_{G}$ justifies the good performances of the adaptation procedure driven only by $\mathcal{M}_{I}$ for a sufficiently fine mesh. As a second check, we verify if the selection of a specific metric does influence the order of convergence of the global error estimator $\eta_{I G}$. The numerical validation shows that no significant difference is detected by selecting a different metric and, for all the choices of $\mathcal{M}$, the order of convergence of the global error estimator is one. In Figure 9, right, we provide an enlarged view of such a comparison, which shows the slight difference among the procedures associated with $\mathcal{M}_{I G}^{\cap}, \mathcal{M}_{I G}^{\max }$, and $\mathcal{M}_{I G}^{\gamma}$.

Robustness of the error estimators. We investigate the robustness of the error estimators $\eta_{I}$ and $\eta_{G}$ by computing the associated effectivity index

$$
\text { E.I. } I=\frac{\eta_{I}}{\left\|f-I_{h} f^{E}\right\|_{L^{1}(\Gamma)}}, \quad \text { E.I. }{ }_{G}=\frac{\eta_{G}}{\left\|d-I_{h} d\right\|_{L^{1}(\Gamma)}},
$$

Copyright $@$ by SIAM. Unauthorized reproduction of this article is prohibited. 
TABLE 4

Effectivity index for the interpolation and the geometric errors for the first test case (left panel) and for the second test case (second panel).

\begin{tabular}{|c|c|c||c|c|c|}
\hline \multicolumn{3}{|c||}{ Test Case 1 } & \multicolumn{3}{c|}{ Test Case 2} \\
\hline Elements & E.I. $I$ & E.I. $G$ & Elements & E.I. $I$ & E.I. $G$ \\
\hline 2920 & 1.6083 & 0.927002 & 4152 & 1.7695 & 0.6102 \\
\hline 6635 & 1.4692 & 0.888572 & 11818 & 1.8502 & 0.6348 \\
\hline 10573 & 1.4105 & 0.851926 & 15506 & 1.6897 & 0.6854 \\
\hline 14240 & 1.4798 & 0.874772 & 22144 & 1.694 & 0.6401 \\
\hline
\end{tabular}
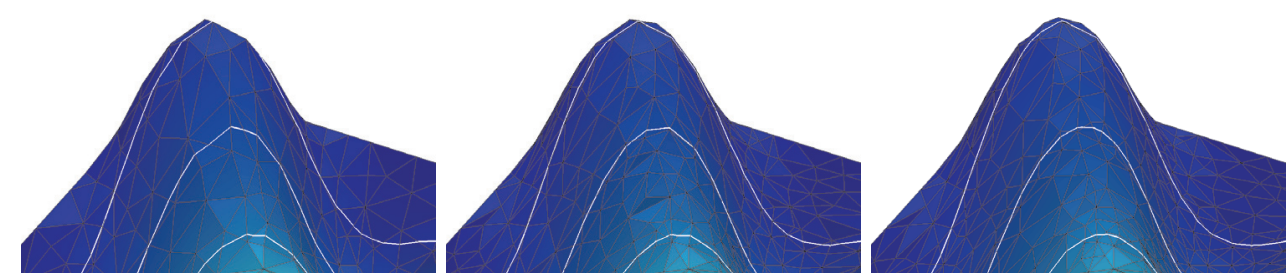

FIG. 10. Test case 1: particular of the function $f_{1}$ on the surface $\Gamma_{1}$ for $\gamma=0.1$ (left), 0.5 (middle), 0.7 (right).

respectively. The optimal value is 1 . Nevertheless, at least a stagnation of the effectivity index is desirable, when the number of the mesh elements increases. Such a stagnation is assessed by Table 4, left panel, for both estimators $\eta_{I}$ and $\eta_{G}$, whose effectivity index settles around 1.4 and 0.8 , respectively. Thus, while the interpolation error estimator slighly overestimates the actual error, an underestimation of the geometric error is provided by $\eta_{G}$.

Sensitivity with respect to $\gamma$. We analyze the sensitivity of the adapted mesh yielded by the metric $M_{I G}^{\gamma}$ with respect to the value of the parameter $\gamma$. To this aim, we fix the tolerance TOL to 2.e-05 and we choose $\gamma=0.1,0.5$, and 0.7 . This choice leads to gradually increasing the contribution of interpolation information in $M_{I G}^{\gamma}$. The selected TOL is sufficiently small to ensure a rather accurate description of the surface, independently of $\gamma$. We focus on the peak at $(1,1)$. Figure 10 depicts the enlarged view of the adapted mesh for the three values of $\gamma$, together with the contour lines of the solution. The gradual inclusion of the interpolation information provides smoother contour lines, together with a slight increase of the mesh cardinality and of the aspect ratio of the elements which are correctly stretched to follow the directionalities of the surface.

Mismatch between $\Gamma_{\boldsymbol{h}}$ and $\boldsymbol{I}_{\boldsymbol{h}} \boldsymbol{d}$. As a last check we furnish numerical support to the statement of Proposition 3.3. To verify that the zero level set of function $I_{h} d$ may be assumed as a surrogate of the discrete surface $\Gamma_{h}$, we compute the value of $I_{h} d$ on the adapted mesh $\Gamma_{h}$ and we check if such a value approaches zero when the surface mesh is gradually adapted. Figure 11 certifies the expected trend. We plot the interpolated distance function $I_{h} d$ on three adapted meshes generated via $\mathcal{M}_{I G}^{\max }$ and consisting of 1643,3792 , and 6051 triangular faces (left to right), respectively. The values assumed by $I_{h} d$ exhibit a more uniform distribution and are closer to zero as the mesh is progressively adapted. Moreover, the maximum value reached by $I_{h} d$ also on the coarsest grid is small (about 8.e-03). This confirms that $I_{h} d$ may be reasonably employed to represent the discrete surface $\Gamma_{h}$. 

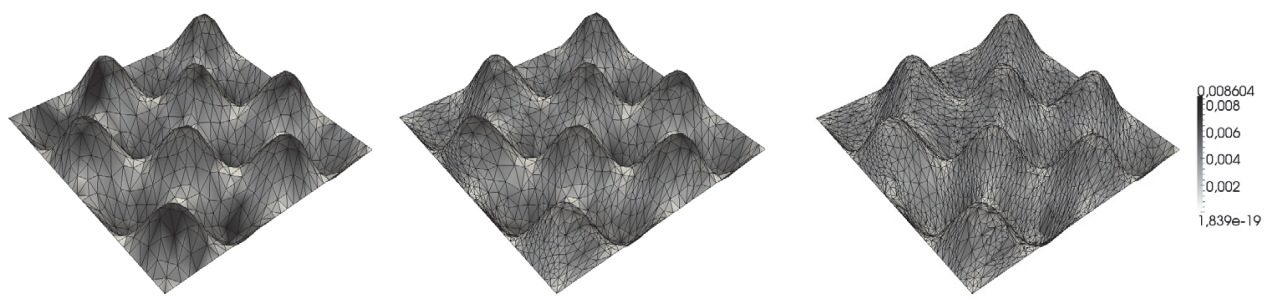

FIG. 11. Test case 1: evaluation of the distance $I_{h} d$ on the adapted mesh $\Gamma_{h}$ for an increasing number of mesh elements (left to right).

5.2.2. Test case 2: A closed surface. Let $\Gamma_{2}$ be the toroidal surface coinciding with the zero level set of the signed distance function $d_{2}:[-1.5,1.5] \times[-1.5,1.5] \times$ $[-0.5,0.5] \rightarrow \mathbb{R}$ such that $d_{2}(\mathbf{x})=\left(0.5-\sqrt{x_{1}^{2}+x_{2}^{2}}\right)^{2}+x_{3}^{2}-1$. On this surface we assign the function $f_{2}: \Gamma_{2} \rightarrow \mathbb{R}$ with $f_{2}(\mathbf{x})=100 \tanh \left(40 x_{1}\right)$ which assumes the two constant values -100 and 100 separated by a steep gradient in correspondence with the two unitary circumferences in the plane $x_{2} O x_{3}$ and centered at $(0,-1,0)$ and $(0,1,0)$, respectively (see Figure 6, right).

On this new configuration we repeat some of the numerical controls performed for the first test case. For this purpose, we run algorithm MeshAdaptation by setting TOL $=5 . \mathrm{e}-03$, maxIter $=10$. Moreover, we fix $\gamma=2 / 3$ for the parameter mixing the interpolation with the geometric information in $\mathcal{M}_{I G}^{\gamma}$.

Comparison among metrics. We collect in Figure 12 the adapted meshes generated by the four metrics $\mathcal{M}_{I G}^{\cap}, \mathcal{M}_{I G}^{\max }, \mathcal{M}_{I G}^{\gamma}$ and $\mathcal{M}_{I}$, respectively. The layer is sharply captured by all four metrics via thin elements correctly oriented (see Figure 13). The coarsest grids are predicted by $\mathcal{M}_{I G}^{\cap}$ (about 7600 triangles) and $\mathcal{M}_{I}$ (about 5100 triangles), whereas the other two meshes are constituted by about 9200 faces. Unlike test case 1 , the limits of the adaptive procedure associated with $\mathcal{M}_{I}$ are evident despite the small tolerance. Indeed, the shape of $\Gamma_{2}$ is badly captured as highlighted by the very irregular surface in Figure 12(d) and by the largest value of $d_{\max }$ in Table 1, right panel.

The intersection metric locates the anisotropic elements essentially in correspondence with the layer and correctly stretches them (see Figure 13(a)). Nevertheless, analogously to the previous test case, the anisotropic features of the corresponding mesh are less meaningful compared with the ones yielded by the other metrics. This is confirmed by the details in Figure 13(b)-(c) as well as by the values of $\sigma_{\max }$ in Table 1, right panel. The details in Figure 14 on the regions where $f_{2}$ reaches the maximum value corroborate that the absence of geometric information in $\mathcal{M}_{I}$ yields a very inaccurate approximation of $\Gamma_{2}$. Moreover, also for this test case metric $\mathcal{M}_{I G}^{\cap}$ generates a more isotropic mesh (see Figure 14(a)).

Anisotropy versus isotropy. We verify the benefits led by an anisotropic mesh adaptation via Tables 5 and 6 . As for the first test case, we exclude from this check the procedure driven by $\mathcal{M}_{I G}^{\cap}$ while computing the errors $e_{\text {tot }}, e_{\text {mean }}, e_{\max }$ in (5.2) for $\mathcal{M}_{I}, \mathcal{M}_{I G}^{\max }, \mathcal{M}_{I G}^{\gamma}$.

In Table 5, the comparison between anisotropy and isotropy is performed for a fixed number of elements. Both the total and the mean error reduce by a factor about equal to one-half and the accuracy of the approximation improves also in terms of maximum error (this trend is not detected in Table 2). If vice versa, we fix the 


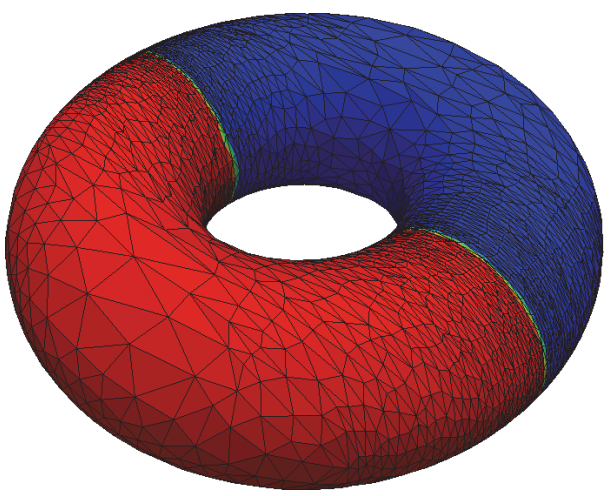

(a)

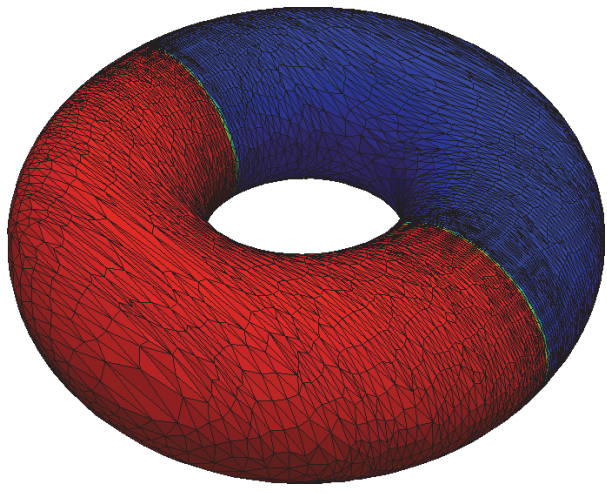

(c)

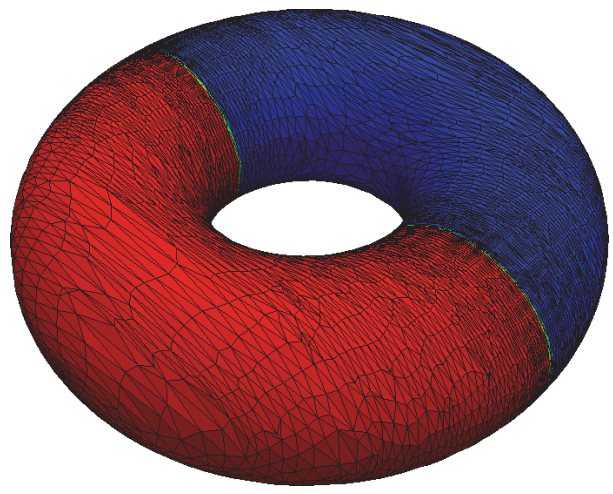

(b)

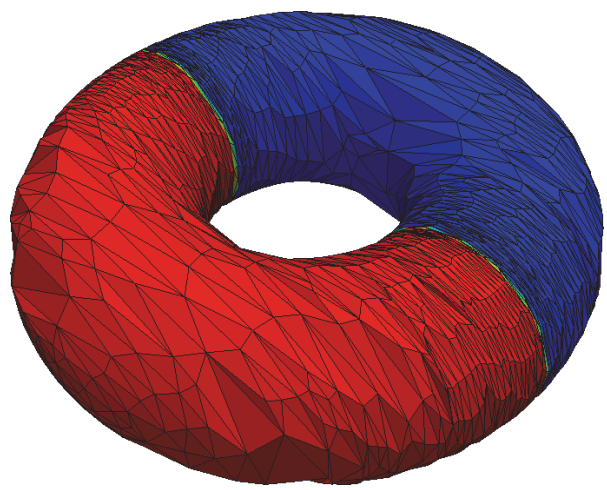

(d)

FIG. 12. Test case 2: adapted mesh $\mathcal{T}_{h}^{A}$ generated via the metric (a) $\mathcal{M}_{I G}^{\cap}$, (b) $\mathcal{M}_{I G}^{\max }$, (c) $\mathcal{M}_{I G}^{\gamma}$, and (d) $\mathcal{M}_{I}$.

accuracy and compare the two strategies in terms of number of elements as in Table 6 , and we get the expected reduction in the anisotropic case, with a factor of one-third for $\mathcal{M}_{I}$ and $\mathcal{M}_{I G}^{\max }$ and of one-half for $\mathcal{M}_{I G}^{\gamma}$.

TABLE 5

Test case 2: anisotropy versus isotropy for a fixed cardinality of the mesh.

\begin{tabular}{c|cc|cc|cc}
\hline & \multicolumn{2}{|c|}{$\mathcal{M}_{I}$} & \multicolumn{2}{c|}{$\mathcal{M}_{I G}^{\max }$} & \multicolumn{2}{c}{$\mathcal{M}_{I G}^{\gamma}$} \\
\hline & Isotropy & Anisotropy & Isotropy & Anisotropy & Isotropy & Anisotropy \\
\hline elements & 3376 & 3640 & 3672 & 3652 & 1548 & 1660 \\
$e_{\text {tot }}$ & $1.901 \mathrm{e}-01$ & $8.884 \mathrm{e}-02$ & $1.864 \mathrm{e}-01$ & $8.887 \mathrm{e}-02$ & $5.820 \mathrm{e}-01$ & $3.463 \mathrm{e}-01$ \\
$e_{\text {mean }}$ & $5.633 \mathrm{e}-05$ & $2.440 \mathrm{e}-05$ & $5.078 \mathrm{e}-05$ & $2.429 \mathrm{e}-05$ & $3.759 \mathrm{e}-04$ & $2.086 \mathrm{e}-04$ \\
$e_{\text {max }}$ & $2.237 \mathrm{e}-03$ & $1.646 \mathrm{e}-03$ & $1.848 \mathrm{e}-02$ & $1.641 \mathrm{e}-03$ & $1.648 \mathrm{e}-02$ & $8.074 \mathrm{e}-03$ \\
\hline
\end{tabular}

Convergence analysis. We analyze the asymptotic trend of both the estimators $\eta_{I}$ and $\eta_{G}$ as a function of $\# \mathcal{T}_{h}$. Figure 15 , left, shows the associated $\log \log$ plot. The interpolation error estimator is characterized by an order of convergence equal to one with respect to $1 /\left(\# \mathcal{T}_{h}\right)$, exactly as in Figure 9 , left. The geometric error estimator converges faster than $\eta_{I}$, with an order very close to two (instead of the order 1.5 as in Figure 9, left). 


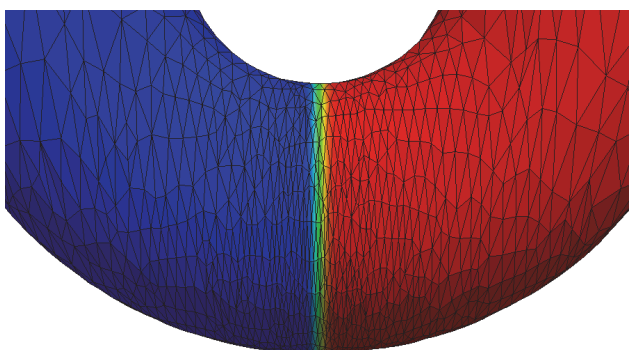

(a)

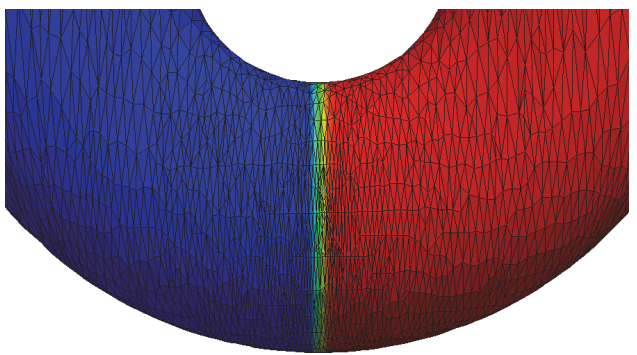

(c)

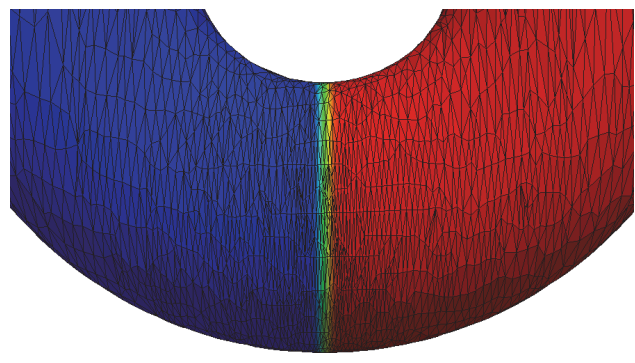

(b)

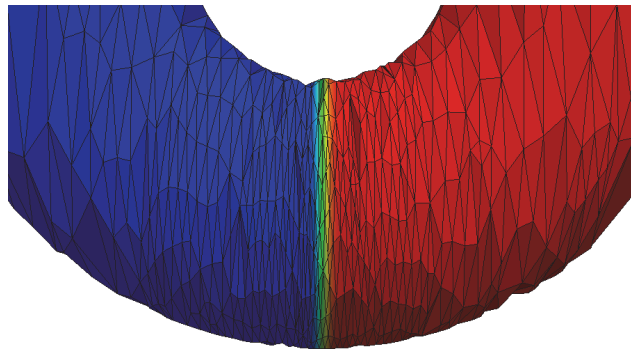

(d)

FIG. 13. Test case 2: detail in correspondence with the layer for the adapted mesh $\mathcal{T}_{h}^{A}$ generated via the metric (a) $\mathcal{M}_{I G}^{\cap}$, (b) $\mathcal{M}_{I G}^{\max }$, (c) $\mathcal{M}_{I G}^{\gamma}$, and (d) $\mathcal{M}_{I}$.

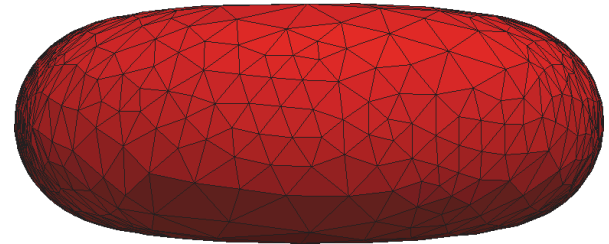

(a)

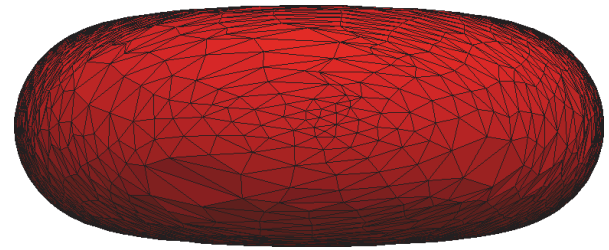

(c)

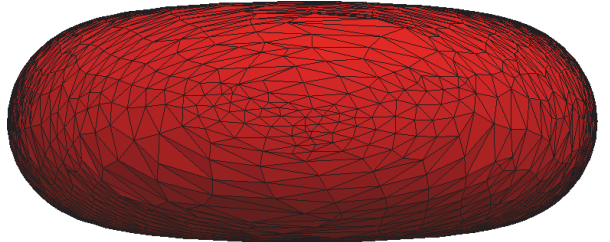

(b)

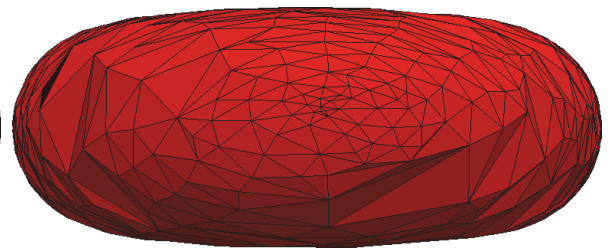

(d)

FIG. 14. Test case 2: detail on the constant part associated with the maximum value of $f_{2}$ for the mesh generated via the metric (a) $\mathcal{M}_{I G}^{\cap}$, (b) $\mathcal{M}_{I G}^{\max }$, (c) $\mathcal{M}_{I G}^{\gamma}$, and (d) $\mathcal{M}_{I}$.

Figure 15, right, confirms the slight sensitivity of the rate of convergence of the global estimator with respect to the metric driving the adaptive procedure by providing a zoom-in on the convergence history of $\eta_{I G}$. Indipendently of the selected metric, we confirm a convergence of the first order.

Robustness of the error estimators. In Table 4, right panel, we compute the effectivity indices in (5.4) on four adapted meshes to check the robustness of the 
TABLE 6

Test case 2: anisotropy versus isotropy for a fixed accuracy of the mesh.

\begin{tabular}{c|cc|cc|cc}
\hline & \multicolumn{2}{|c|}{$\mathcal{M}_{I}$} & \multicolumn{2}{c|}{$\mathcal{M}_{I G}^{\max }$} & \multicolumn{2}{c}{$\mathcal{M}_{I G}^{\gamma}$} \\
\hline & Isotropy & Anisotropy & Isotropy & Anisotropy & Isotropy & Anisotropy \\
\hline elements & 11030 & 4732 & 11030 & 4746 & 8162 & 4084 \\
$e_{\text {tot }}$ & $6.327 \mathrm{e}-02$ & $6.490 \mathrm{e}-02$ & $6.327 \mathrm{e}-02$ & $6.420 \mathrm{e}-02$ & $8.656 \mathrm{e}-02$ & $8.733 \mathrm{e}-02$ \\
$e_{\text {mean }}$ & $5.736 \mathrm{e}-06$ & $1.371 \mathrm{e}-05$ & $5.736 \mathrm{e}-06$ & $1.352 \mathrm{e}-05$ & $1.060 \mathrm{e}-05$ & $2.138 \mathrm{e}-05$ \\
$e_{\max }$ & $4.212 \mathrm{e}-04$ & $1.019 \mathrm{e}-03$ & $4.212 \mathrm{e}-04$ & $1.019 \mathrm{e}-03$ & $7.884 \mathrm{e}-04$ & $1.775 \mathrm{e}-03$ \\
\hline
\end{tabular}

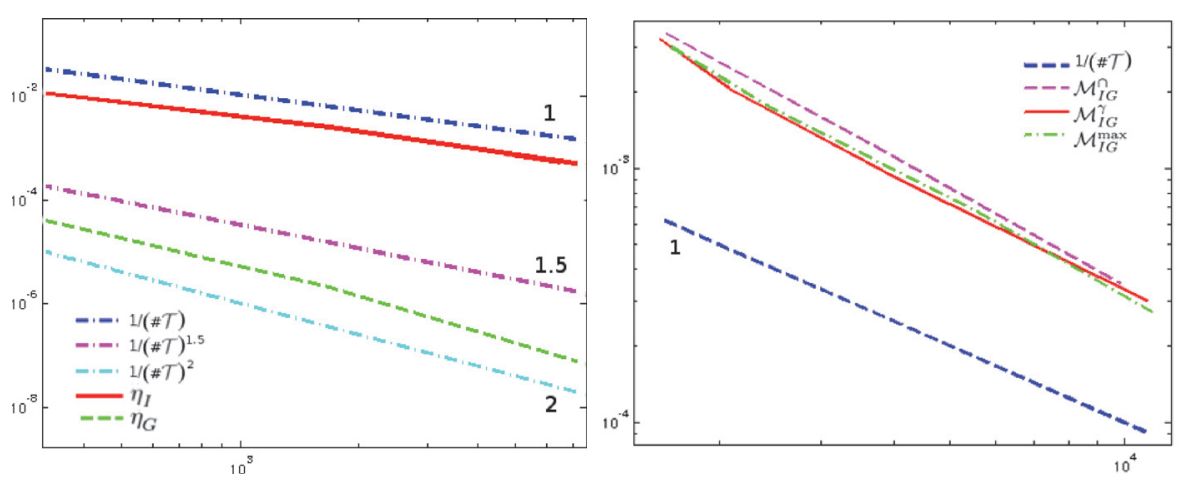

FIG. 15. Test case 2: convergence history for the estimators $\eta_{I}$ (solid line) and $\eta_{G}$ (dashed line) in a loglog plot (left); convergence trend of the global error estimator for three different metrics in a loglog plot (right).

interpolation and the geometric error estimators. A sort of stagnation is detected for both indices, around the values 1.7 and 0.6 , respectively. This means that an overestimation of $\eta_{I}$ and an underestimation of $\eta_{G}$ take place, with a more emphatic trend with respect to the first test case.

6. Conclusions and future developments. We have proposed a new approach for an anisotropic control of the error related to the approximation of an $H^{1}(\Gamma)$-function via a piecewise linear quasi-interpolant operator on a surface $\Gamma \subset \mathbb{R}^{3}$ implicitly defined. The analysis automatically takes into account the approximation of the function and the fitting of the surface, thus leading to a unique adaptive procedure able to simultaneously match the directionalities of $f$ and $\Gamma$. The numerical validation in section 5.2 confirms the robustness of the adaptive tool. The expected advantages with respect to a standard isotropic mesh adaptation are verified on both open and closed surfaces. The convergence analysis corroborates the results expected from the theory, i.e., an order of convergence equal to one for the interpolation error and a higher order for the geometric contribution, independently of the selected global metric. Concerning the robustness of the separate error estimators, the values of the corresponding effectivity index highlight a slight overestimation for the interpolaton estimator, whereas the geometric estimator underestimates the actual error.

The promising results of this work suggest as a next step the development of a corresponding a posteriori error analysis, possibly in a goal-oriented setting [4, 22].

\section{REFERENCES}

[1] F. Alauzet, A. Loseille, A. Dervieux, and P. Frey, Multi-dimensional continuous metric for mesh adaptation, in Proceedings of the 15th International Meshing Roundtable, P. P. Pebay, ed., Springer-Verlag, Berlin, 2006, pp. 191-214. 
[2] T. Apel, Anisotropic Finite Elements: Local Estimates and Applications, in Advances in Numerical Mathematics, Teubner, Stuttgart, 1999.

[3] T. Apel And C. Pester, Clément-type interpolation on spherical domains: Interpolation error estimates and application to a posteriori error estimation, IMA J. Numer. Anal., 25 (2005), pp. 310-336.

[4] R. BECKER AND R. RANNACHER, An optimal control approach to a posteriori error estimation in finite element methods, Acta Numer., 10 (2001), pp. 1-102.

[5] A. Belme, A. Dervieux, And F. Alauzet, Time accurate anisotropic goal-oriented mesh adaptation for unsteady flows, J. Comput. Phys., 231 (2012), pp. 6323-6348.

[6] H. Borouchaki, P. Laug, And P. L. George, Parametric surface meshing using a combined advancing-front generalized Delaunay approach, Internat. J. Numer. Methods Engrg., 49 (2000), pp. 233-259.

[7] R. Boussetta, T. Coupez, And L. Fourment, Adaptive remeshing based on a posteriori error estimation for forging simulation, Comput. Methods Appl. Mech. Engrg., 195 (4849) (2006), pp. 6626-6645.

[8] M. J. Castro-Diaz, F. Hecht, B. Mohammadi, and O. Pironneau, Anisotropic unstructured mesh adaptation for flow simulations, Internat. J. Numer. Methods Fluids, 25 (1997), pp. 475-491.

[9] Ph. Clément, Approximation by finite element functions using local regularization, RAIRO Anal. Numer., 2 (1975), pp. 77-84.

[10] T. Coupez, Metric construction by length distribution tensor and edge based error for anisotropic adaptive meshing, J. Comput. Phys., 230 (2011), pp. 2391-2405.

[11] F. Dassi, B. Ettinger, S. Perotto, and L. M. Sangalli, A mesh simplification strategy for a spatial regression analysis over the cortical surface of the brain, Appl. Numer. Math., 90 (2015), pp. 111-131.

[12] A. Demlow and G. Dziuk, An adaptive finite element method for the Laplace-Beltrami operator on implicity defined surfaces, SIAM J. Numer. Anal., 45 (2007), pp. 421-442.

[13] G. Dziuk and C. M. Elliott, Finite element methods for surface PDEs, Acta Numer., 22 (2013), pp. 289-396.

[14] H. Edelsbrunner, Geometry and Topology for Mesh Generation, Cambridge University Press, Cambridge, UK, 2001.

[15] P. E. Farrell, S. Micheletti, and S. Perotto, An anisotropic Zienkiewicz-Zhu type error estimator for $3 D$ applications, Internat. J. Numer. Methods Engrg., 85 (2011), pp. 671-692.

[16] F. Fierro And A. Veeser, A posteriori error estimators, gradient recovery by averaging, and superconvergence, Numer. Math., 103 (2006), pp. 267-298.

[17] L. Formaggia and S. Perotto, New anisotropic a priori error estimates, Numer. Math., 89 (2001), pp. 641-667.

[18] P. J. Frey and H. Borouchaki, Geometric surface mesh optimization, Comput. Vis. Sci., 1 (3) (1998), pp. 113-121.

[19] P. J. Frey and H. Borouchaki, Surface meshing using a geometric error estimate, Internat. J. Numer. Methods Engrg., 50 (2003), pp. 227-245.

[20] P. L. George And H. Borouchaki, Delaunay Triangulation and Meshing-Application to Finite Element, Editions Hermes, Paris, 1998.

[21] E. H. Georgoulis, E. Hall, And P. Houston, Discontinuous Galerkin methods for advectiondiffusion-reaction problems on anisotropically refined meshes, SIAM J. Sci. Comput., 30 (2007), pp. 246-271.

[22] M. B. Giles And E. SüLI, Adjoint methods for PDEs: A posteriori error analysis and postprocessing by duality, Acta Numer., 11 (2002), pp. 145-236.

[23] G. H. Golub and C. F. Van Loan, Matrix Computations, Johns Hopkins University Press, Baltimore, 1996.

[24] E. Hartmann, On the curvatures of curves and surfaces defined by normal forms, Comput. Aided Geom. Design, 16 (1999), pp. 355-376.

[25] M. Holst, Adaptive numerical treatment of elliptic systems on manifolds, Adv. Comput. Math., 15 (2001), pp. 139-191.

[26] H. Hoppe, T. DeRose, T. Duchamp, J. McDonald, and W. Stuetzle, Mesh optimization, in Proceedings of the 20th Annual Conference on Computer Graphics and Interactive Techniques, SIGGRAPH'93, ACM Press, New York, 1993.

[27] W. Huang, Metric tensors for anisotropic mesh generation, J. Comput. Phys., 204 (2005), pp. 633-665.

[28] C. L. Lawson, Software for $C^{1}$ surface interpolation, in Mathematical Software III, J. R. Rice, ed., Academic Press, New York, 1977, pp. 161-194.

Copyright $@$ by SIAM. Unauthorized reproduction of this article is prohibited. 
[29] S. Micheletti and S. Perotto, Output functional control for nonlinear equations driven by anisotropic mesh adaptation: The Navier-Stokes equations, SIAM J. Sci. Comput., 30 (2008), pp. 2817-2854.

[30] P. Morin And R. Nochetto, AFEM for the Laplace-Beltrami operator on graphs: Design and conditional contraction property, Math. Comp., 80 (2011), pp. 625-648.

[31] J. Pellerin, B. Lévy, G. Caumon, and A. Botella, Automatic surface remeshing of $3 d$ structural models at specified resolution: A method based on Voronoi diagrams, Comput. Geosci., 62 (2014), pp. 103-116.

[32] R. Ronfard and J. Rossignac, Full-range approximation of triangulated polyhedra, Comput. Graphics Forum, 15 (1996), pp. 67-76.

[33] E. Sauvage, J. F. Remacle, and E. Marchandise, Metric field construction for anisotropic mesh adaptation with application to blood flow simulations, Int. J. Numer. Methods Biomed. Eng., 30 (2014), pp. 1326-1346.

[34] R. SCHNEIDER, Towards practical anisotropic adaptive FEM on triangular meshes: A new refinement paradigm, Comput. Vis. Sci., 15 (2012), pp. 247-270.

[35] L. R. SсотT AND S. Zhang, Finite element interpolation of non-smooth functions satisfying boundary conditions, Math. Comp., 54 (1990), pp. 483-493.

[36] D. A. Venditti AND D. L. Darmofal, Anisotropic grid adaptation for functional outputs: Application to two-dimensional viscous flows, J. Comput. Phys., 187 (2003), pp. 22-46.

[37] R. VERfürth, A Review of a Posteriori Error Estimation and Adaptive Mesh-Refinement Techniques, Wiley-Teubner, New York, 1996.

Copyright $@$ by SIAM. Unauthorized reproduction of this article is prohibited. 